\title{
SMOS INSTRUMENT PERFORMANCE AND CALIBRATION AFTER 5 YEARS IN ORBIT
}

M. Martín-Neira (1), R. Oliva (2), I. Corbella (3), F. Torres (3), N. Duffo (3), I. Durán (3), J. Kainulainen (4), J. Closa (5), A. Zurita (5), F. Cabot (6), A. Khazaal (6), E. Anterrieu (7), J. Barbosa (8), G. Lopes (8), J. Tenerelli (9), R. Díez-García (10), J. Fauste (2), F. Martín-Porqueras (11), V. González-Gambau (12), A. Turiel (12), S.

9 Delwart (13), R. Crapolicchio (13), M. Suess (1)

\section{Corresponding author}

M. Martín-Neira and M. Suess are with ESA-ESTEC, Keplerlaan 1, 2200 AG Noordwijk, The Netherlands. (e-mail: manuel.Martín-Neira@esa.int)

\section{Authors' affiliations}

(1) European Space Agency at ESTEC, Noordwijk, The Netherlands.

(2) European Space Agency at ESAC, Villanueva de la Cañada, Spain.

(3) Polytechnic University of Catalonia (UPC), Barcelona, Spain.

(4) Harp Technologies Ltd., Espoo, Finland.

(5) EADS-CASA Espacio, Madrid, Spain.

(6) Centre d'Etudes Spatiales de la BIOsphère (CESBIO), Toulouse, France.

(7) Institut de Recherche en Astrophysique et Planétologie (IRAP), Toulouse, France.

(8) DEIMOS, Lisbon, Portugal.

(9) OceanDataLab, Brest, France.

(10) IDEAS, ESAC, Villanueva de la Cañada, Spain.

(11) VEGA Telespazio, ESAC, Villanueva de la Cañada, Spain.

(12) SMOS Barcelona Expert Centre, Barcelona, Spain.

(13) European Space Agency at ESRIN, Frascati, Italy.

\section{Keywords}

Soil Moisture and Ocean Salinity (SMOS) Mission, soil moisture, sea surface salinity, L-Band radiometry, aperture synthesis, MIRAS 
REPLACE THIS LINE WITH YOUR PAPER IDENTIFICATION NUMBER (DOUBLE-CLICK HERE TO EDIT) <

\section{Abstract}

42 ESA's Soil Moisture and Ocean Salinity (SMOS) mission, launched 2-Nov-2009, has been in orbit for over 5 43 years, and its Microwave Imaging Radiometer with Aperture Synthesis (MIRAS) in two dimensions keeps 44 working well. The calibration strategy remains overall as established after the commissioning phase, with a few 45 improvements. The data for this whole period has been reprocessed with a new fully polarimetric version of the 46 Level-1 processor which includes a refined calibration schema for the antenna losses. This reprocessing has 47 allowed the assessment of an improved performance benchmark. An overview of the results and the progress 48 achieved in both calibration and image reconstruction is presented in this contribution.

\section{INTRODUCTION}

With an experience of over 5 years of in-orbit operation, much has been learnt on how MIRAS works and how its observations can be improved through better calibration and image reconstruction techniques. The purpose of this paper is to update the reader with the latest results on the payload performance and data processing of the SMOS mission (Mecklenburg et al., 2012). SMOS is currently delivering several products, some of them used by operational systems, others only for scientific research (Mecklenburg et al., in press). MIRAS is a Microwave Imaging Radiometer with two-

57 dimensional Aperture Synthesis, which remains being the first and so far, the only one of its kind, in 58 space. The main feature of MIRAS is that it obtains two-dimensional images at every snapshot without 59 needing any mechanical scanning of its antenna, a very distinct capability when compared with

60 traditional scanners or push-broom radiometers. A detailed description of the instrumental aspects of 61 MIRAS can be found in (McMullan et al., 2008) while the on-board Calibration System and respective 62 in-flight calibration strategy are described in (Brown et al., 2008) and (Martín-Neira et al., 2008). One 63 year after launch the calibration approach was slightly modified with the initial flight experience, and 64 the first SMOS instrument in-orbit performance was assessed in (Oliva et al., 2013), including the 65 effect of the unexpectedly severe Radio Frequency Interference from ground transmitters (Oliva et al., 66 2012). The present paper will then follow the same structure as (Oliva et al., 2013), with important 


\section{REPLACE THIS LINE WITH YOUR PAPER IDENTIFICATION NUMBER (DOUBLE-CLICK}

HERE TO EDIT) <

67 additions brought by the accumulated experience of over 5 years: Section 3 provides an overview of the 68 main sources of error and the current mitigation strategies used to overcome them; Section 4 69 summarizes the current status of calibration activities, including all latest modifications to the initial 70 calibration plan; Section 5 presents the in-orbit behaviour of the most critical instrument parameters; 71 Section 6 gives the performance obtained with the latest version of the Level-1 processor, through the 72 spatial and temporal analysis of brightness temperature images, and finally, Section 7 includes a view 73 on the current investigations that should lead to the next version of the Level-1 processor with a hint on 74 the expected improvements.

75 It is worth mentioning that, at the time of the writing of this paper, the running version of the 76 operational SMOS Level-1 data processor is V620, that a new version, V700, has been delivered and is 77 under assessment, and that the entire data record of the SMOS mission (from January 2010 onwards) 78 has been reprocessed with V620 and is available to the whole SMOS user community.

\section{ERROR SOURCES AND MITIGATION TECHNIQUES}

\subsection{Error Sources}

82 Different error sources cause different effects on the SMOS brightness temperature images. Therefore 83 in this section the error sources will be presented according to the effect they produce in the images.

\section{4}

\subsubsection{Systematic Spatial Ripple}

Figure 1 presents the deviation, with respect to a forward model, of an image of the brightness temperature measured by SMOS over a portion of the South-Eastern Pacific Ocean in X-polarization (X-polarization refers to the image formed with the signal collected by the horizontal probe of MIRAS antenna elements). The comparison is performed after averaging many snapshots so that random errors induced by the radiometric resolution can be neglected, and only systematic errors remain. The most prominent features of such deviation image are a $+0.96 \mathrm{~K}$ bias and a $1.5 \mathrm{~K}$ rms spatial ripple, both statistics evaluated within the dashed circle shown in Figure 1. Similar statistics can be computed for 


\section{REPLACE THIS LINE WITH YOUR PAPER IDENTIFICATION NUMBER (DOUBLE-CLICK HERE TO EDIT) <}

93 the Y-polarization as well as for the Stokes-3 and Stokes-4 parameters, obtaining, in general, different

94 values for the different parameters, values which, in turn, depend on the particular image reconstruction 95 approach being applied, that is, on the Level-1 processor version. Furthermore, and although it is not as easy to show as with measurements of the relatively uniform ocean, bias and ripples also appear in

97 images acquired over any region of the Earth, be it land, ice or coastlines, and over the Cold Sky, 98 exhibiting a magnitude which is scene-dependent. Interpreting the bias as a spatial ripple of an infinite 99 spatial wavelength, both bias and spatial ripple shall be understood as comprised within the 'spatial ripple' referred to in what follows.

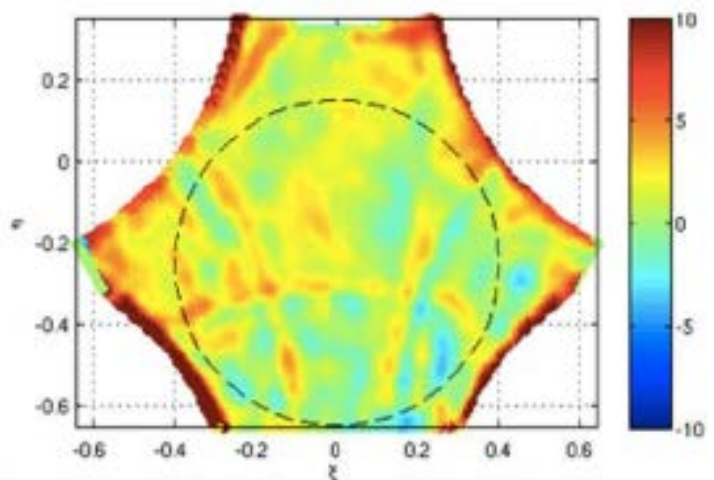

Figure 1: Example of bias and spatial ripples of SMOS images when compared to a radiative transfer ocean model. The axes are the director cosines and the colour scale is in Kelvin.

The cause and existence of the systematic spatial ripple underlying all SMOS images was already studied before SMOS launch (Camps et al., 2005)(Anterrieu, 2007). Thanks to the investigations conducted during the last 5 years in several directions, using flight data, an important conclusion has been consolidated: the spatial ripple results mostly from the combination of having different antenna element patterns and imaging in alias conditions (that is, using a spatial sampling which leads to having aliased images in some parts of the real space). This is illustrated in Figure 2, which shows a similar deviation image to Figure 1 obtained simulating different conditions: the left and right columns assume identical and different - perfectly known- antenna patterns respectively, while the rows correspond to different antenna element spacings, the one of MIRAS $(0.875 \lambda)$ in the top, and another one which is alias-free $(0.55 \lambda)$ in the bottom. As it is evident, the spatial ripple appears only in the top right plot, that 


\section{REPLACE THIS LINE WITH YOUR PAPER IDENTIFICATION NUMBER (DOUBLE-CLICK HERE TO EDIT) <}

117 is, for alias condition and different antenna patterns. If there is no aliasing, or/and if the antenna

118 patterns are all identical, then there is no significant spatial ripple.

119 As a consequence of this, two further results have now been well established: first, even in the ideal

120 case where the antenna pattern of every antenna element were known perfectly well, a non-negligible

121 systematic spatial error would still be present in SMOS images, dubbed 'noise floor'; second, the part

122 of the scene outside the alias-free area does have an impact on the scene recovered in the alias-free

123 region, or in other words, the spatial ripple in the alias-free area depends on the scene in the aliased 124 regions (Corbella et al., 2014).

125 The first result might be the most striking one, since for long, it had been expected that the accurate 126 knowledge of the antenna patterns, very carefully characterized on ground and used in the image 127 reconstruction, would have enabled the acquisition of images with negligible ripple. It also emphasizes 128 the need of having the interferometric instrument with either an alias-free element spacing or as similar 129 antenna patterns as possible, to suppress the spatial ripple from the images. The second result, on the 130 other hand, has been the basis to build ripple reduction methods to improve the current SMOS images, 131 as will be seen in the section devoted to mitigation techniques below. Finally the contribution to the 132 spatial ripple due to the limited knowledge of the antenna patterns and residual calibration errors shall 133 not be forgotten. 

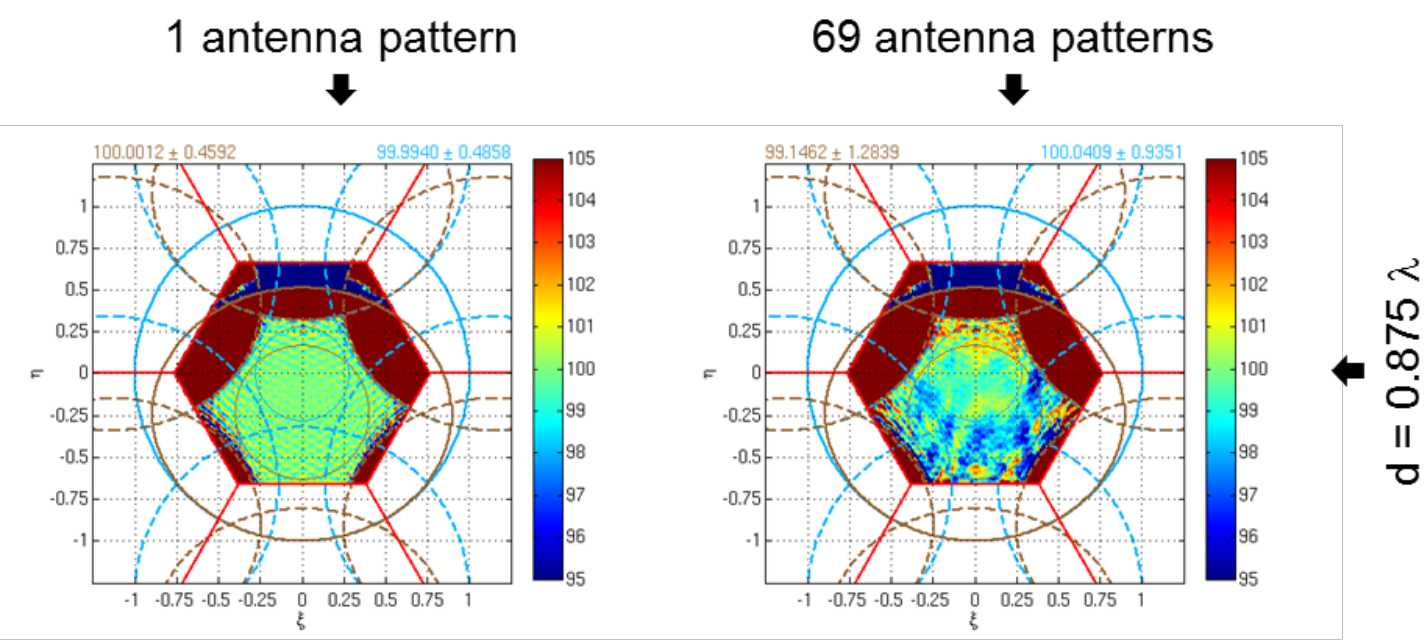

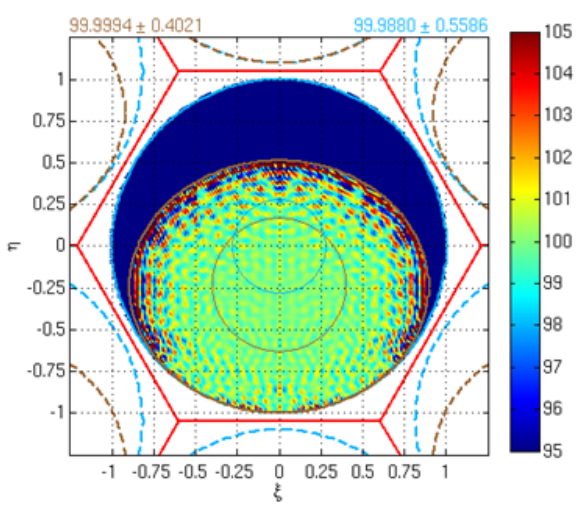

\subsubsection{Sun and RFI Tails}

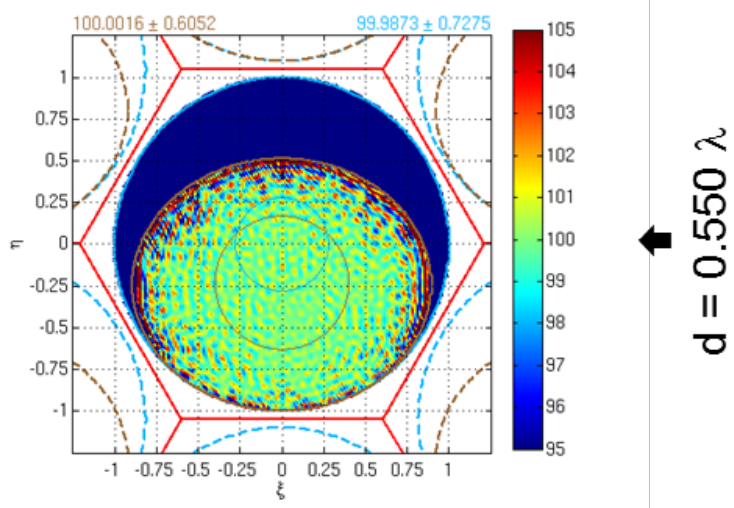

Figure 2: Illustration that the spatial ripple results from the combination of different antenna patterns and alias condition. The image shows the Earth and the sky at a spatially uniform but different brightness temperature, viewed with the nominal SMOS geometry. The axes are the director cosines and the colour bar represents the retrieved brightness temperature in Kelvin.

Figure 3 is a deviation image as Figure 1 but acquired at a time when the Sun is in front of the antenna.

The Sun can be seen as a white circle towards the right side of the unity circle, which represents the front hemisphere of the antenna. The area shaded in blue is the locus of possible positions of the Sun as seen from the SMOS orbit around the year. The Sun locus is sufficiently far away from the extended field of view of SMOS that it would not cause any ripples if it were not because of the aliasing and the side lobes. Indeed, a replica of the Sun is clearly visible inside the field of view. Moreover, the real Sun and its replica appear connected by lines of side lobes which cross the entire field of view, including the alias free region. Extending the lines of side lobes through the black dashed lines it is possible to 


\section{REPLACE THIS LINE WITH YOUR PAPER IDENTIFICATION NUMBER (DOUBLE-CLICK HERE TO EDIT) <}

152 located a second alias in the upper part of the unity circle, outside the extended alias-free field of view.

153 The cause of these side lobes is the same as that of the spatial ripple: the differences across antenna 154 patterns enhance the side lobes joining the Sun aliases, which are generated by the element spacing.

155 Patterns of side lobes are also excited by Radio Frequency Interference (RFI) transmitters, which 156 behave within the image reconstruction process, much in the same way as the Sun. This is illustrated in 157 Figure 4, where the extended alias-free field of view is projected on ground at a location of an RFI 158 source: an hexagonal pattern of side lobes is clearly visible. RFI sources remain being an important 159 error source in SMOS (Oliva et al., in press).

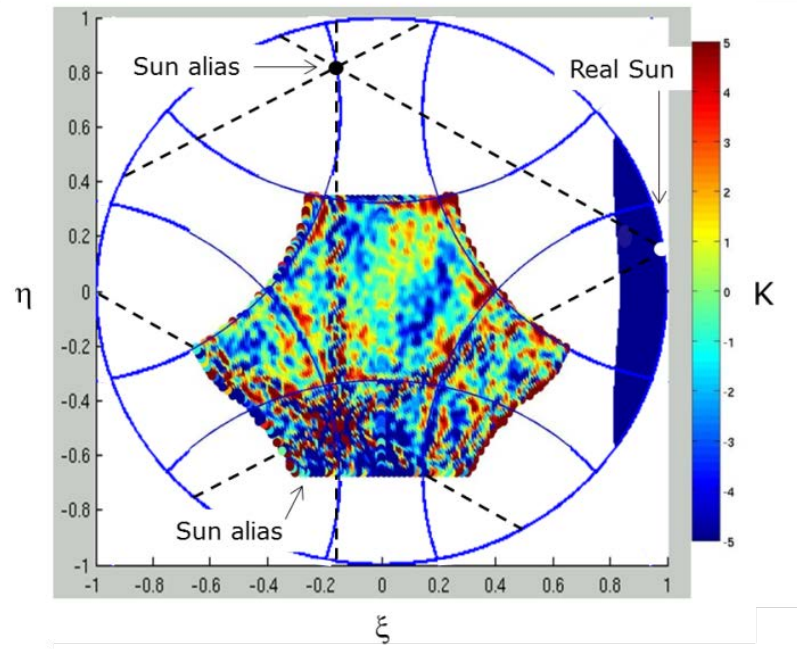

Figure 3: Sun tails and alias affecting an ocean image

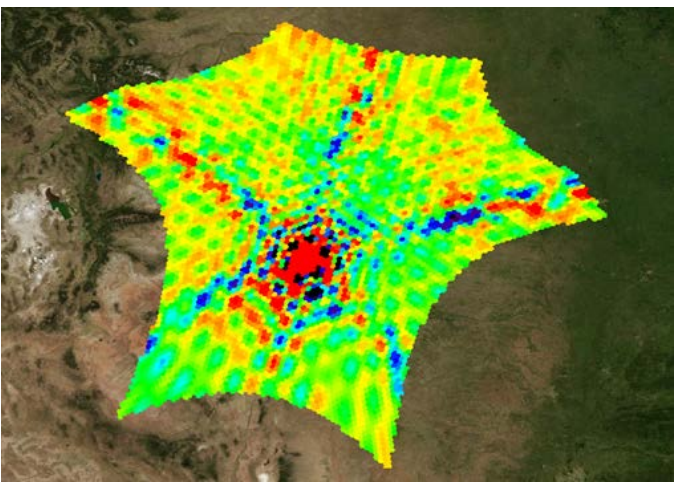

Figure 4: Hexagonal pattern of side lobes excited by a strong Radio Frequency Interference source

\subsubsection{Land-Sea Contamination}


REPLACE THIS LINE WITH YOUR PAPER IDENTIFICATION NUMBER (DOUBLE-CLICK HERE TO EDIT) <

170 The left plot of Figure 5 shows a global view of the oceans with the accumulated deviation of SMOS

171 measurements from a forward model. Brightness temperature warmer than the model are seen around

172 all continental masses which wrongly lead to fresher water retrievals. This feature of SMOS images is

173 referred to as 'land-sea contamination' and is of concern among the Sea Surface Salinity community

174 because it can extend several hundreds of kilometers into the open ocean. Much effort has been devoted

175 to understand the reason behind this land-sea contamination. The current understanding is that it is

176 caused by several contributions. The most important one seems to be a calibration error, of the order of

$1772 \%$, in the amplitude of the correlations, the so-called $\mathrm{G}_{\mathrm{kj}}$ correlator efficiency coefficients (Corbella et

178 al., in press). Although this problem with the correlation coefficients has been identified, to date, the

179 root cause has not been found yet and the search within the instrumental details related to it continues.

180 The second contributor is the spatial ripple described above, generated by the warmer land and 181 extending into the ocean.
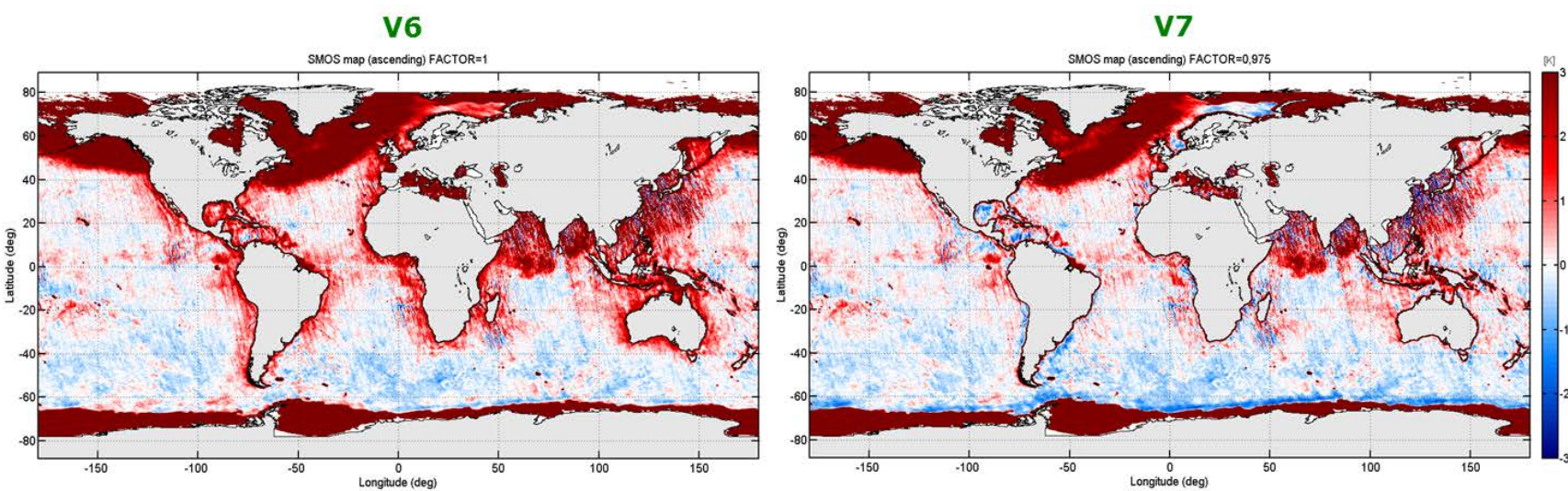

Figure 5: Stokes-1/2 residual images against a radiative transfer ocean model using present correlation efficiency factors (left), where the land-sea contamination is clearly observed around the continental masses, and using corrected values (right), with significantly reduced errors. The warm areas around Alaska, Greenland, Arabian Sea, Gulf of Bengal and Sea of China are due to Radio Frequency Interference, and the blue rim around Antarctica is due to un-modelled sea ice.

\subsubsection{Seasonal Variations}

The right plot of Figure 6 shows the current deviation of the Stokes-1/2 parameter over the Pacific Ocean, averaged within the alias-free field of view, with respect to an ocean model, along the descending passes. The plot spans 5 years, from 2010 till 2014 , and from $60^{\circ}$ South to $60^{\circ}$ North in 


\section{REPLACE THIS LINE WITH YOUR PAPER IDENTIFICATION NUMBER (DOUBLE-CLICK HERE TO EDIT) <}

195 latitude, with a brightness temperature scale of $\pm 1.2 \mathrm{~K}$. This Hovmöller plot constitutes a powerful tool

to analyze any seasonal (and latitudinal) variations. Besides the red stripe during the Commissioning

197 Phase in early 2010, the variations are contained within $\pm 0.4 \mathrm{~K}$ except for the eclipse periods (mid-

198 November to mid-February) and a region around October where some warm signatures are observed.

199 During an eclipse, the Sun, which can be as high as $31^{\circ}$ above the antenna horizon, is suddenly 200 occulted by the Earth. The antenna skin temperature falls down a couple of tens of degrees, from 201 around $28^{\circ} \mathrm{C}$ to some $5^{\circ} \mathrm{C}$. When the satellite exists the Earth shadow, the Sun warms up the antenna 202 again until it reaches the temperature it would have if there had been no eclipse. This post-eclipse 203 transient causes, in every descending orbit, a warm anomaly in the brightness temperature which 204 extends to latitudes as low as $30^{\circ}$ North. On the other hand, the reason for the October warm anomaly 205 has not yet been uncovered. Attempts to correlate it with residual galactic noise or other geophysical signatures have failed and hence, an instrumental origin should be assumed. Furthermore this anomaly seems more intense in 2014.

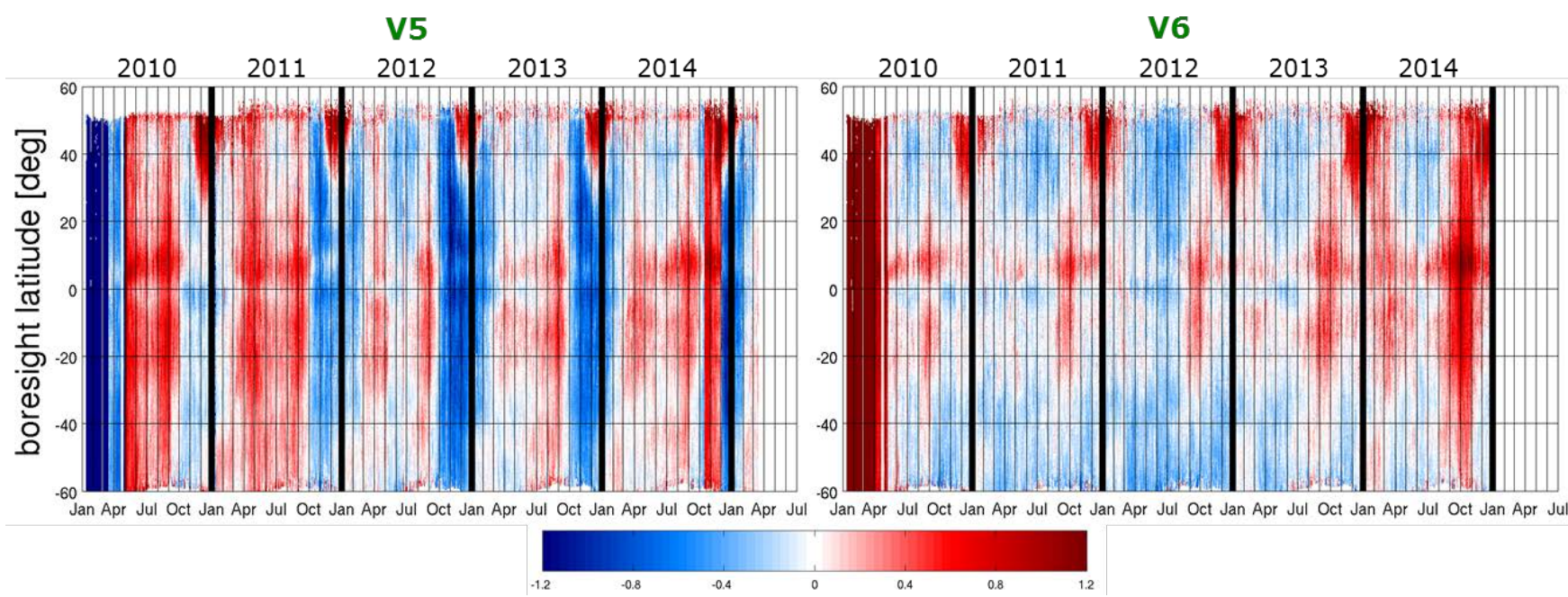

Figure 6: Latitude-Time Hovmöller plot of the descending pass Stokes-1/2deviation from an ocean forward model, averaged in the alias-free area, with V505 (left) and V620 (right) Level 1 processor versions. Colour bar is given in Kelvin. 
REPLACE THIS LINE WITH YOUR PAPER IDENTIFICATION NUMBER (DOUBLE-CLICK HERE TO EDIT) $<$

216 The top right plot of Figure 7 shows the current difference between descending and ascending passes

217 of the Stokes-1/2 parameter over the Pacific Ocean, averaged within the alias-free field of view. The

218 Stokes-1/2 parameters of each pass are first corrected for significant forward model

219 contributions which range from tens of Kelvin (sea surface physical temperature and emissivity)

220 to just a few Kelvin (reflected cold sky, galactic glint, direct and reflected Sun, atmospheric up

221 and down welling). This correction is needed to separate geophysical from instrumental effects as

222 much as possible. The plot span in latitude and time, as well as the brightness temperature scale, are

223 the same as those of Figure 6. The difference between the two passes, shown in the bottom panel, is

224 within $0.8 \mathrm{~K}$ peak-to-peak, the maximum departure, of about $+0.5 \mathrm{~K}$, happening around October,

225 which is the period when the galactic glint is the strongest. There is evidence that such deviation

226 in October is very likely related to a mis-modeling of the galactic glint. It is worth mentioning that

227 the descending minus ascending difference does not show any particular increase in 2014 as seen in the

228 descending pass of Figure 6, meaning that both passes follow each other's variations also in 2014. The

229 top right panel of Figure 7 also shows a clear impact of the eclipse period in latitudes above $30^{\circ}$

230 North. 
REPLACE THIS LINE WITH YOUR PAPER IDENTIFICATION NUMBER (DOUBLE-CLICK

HERE TO EDIT) $<$

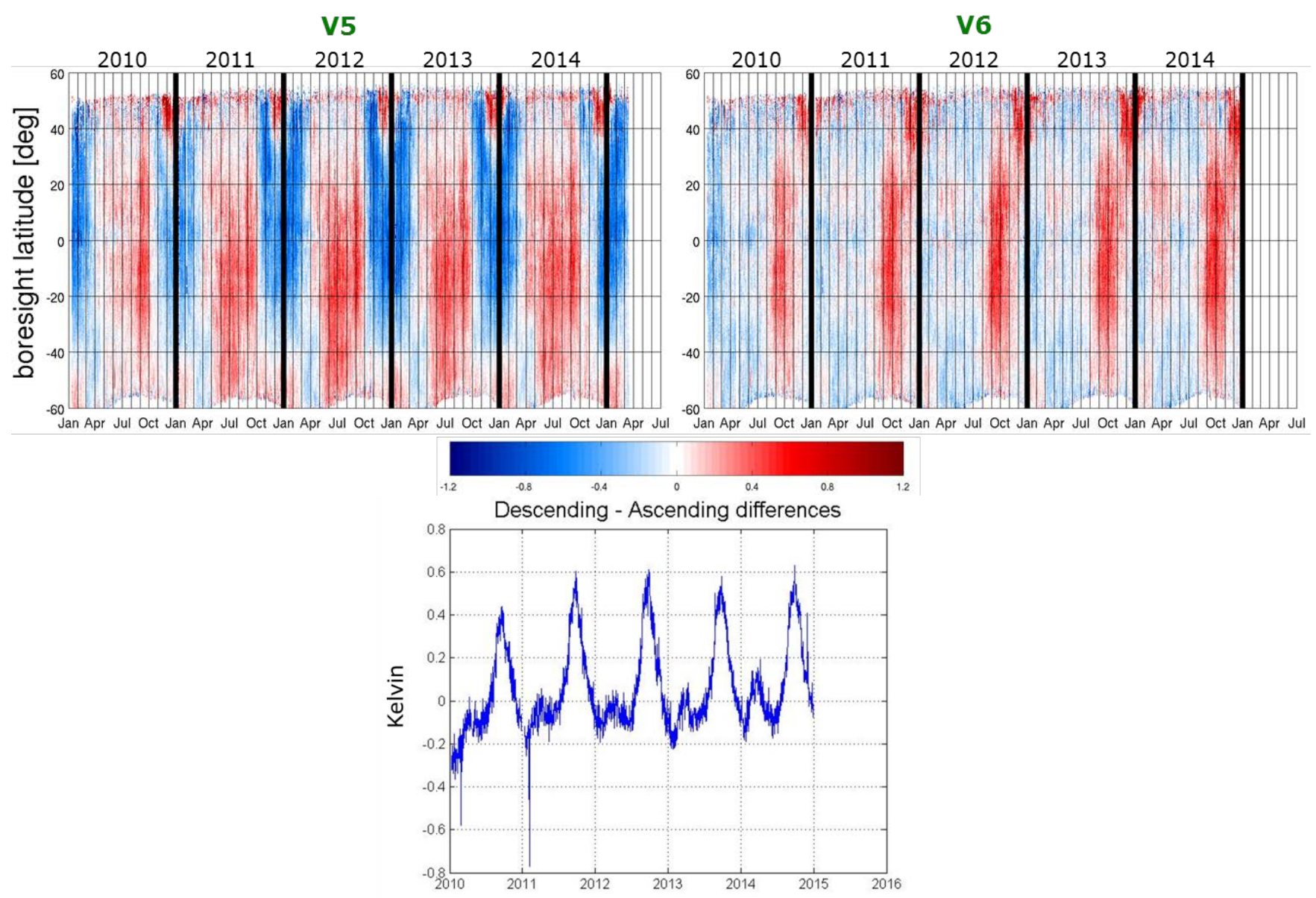

Figure 7: Descending-minus-Ascending pass Stokes-1/2 parameter over the Pacific Ocean, averaged in the alias-free area, with V505 (top left) and V620 (top right) Level 1 processor versions. Colour bar is given in Kelvin. Same parameter averaged from $40^{\circ}$ South to $5^{\circ}$ North to avoid RFI and eclipse effects (bottom)

\subsection{Error Mitigation Techniques}

241 This section presents a summary of the techniques which have been attempted to mitigate, with more or

242 less success, the various error sources described in the previous section. The overall approach to 243 mitigate any of the errors has been to first understand the mechanism causing them and to then build a 244 new calibration or image reconstruction approach to reduce it. Empirical corrections have been avoided 245 as much as possible, and when accepted, they have been applied just once for all 5 year data set. In this 246 section the focus shall be in image reconstruction based solutions, leaving for a later section those 247 improvements brought into the new in-orbit calibration plan. 
REPLACE THIS LINE WITH YOUR PAPER IDENTIFICATION NUMBER (DOUBLE-CLICK HERE TO EDIT) <

\subsubsection{Correction of Systematic Spatial Ripple}

As explained earlier, given an antenna element spacing and a level of dissimilarity between the patterns of these, there is a minimum spatial ripple, the noise floor, which cannot be avoided. From a purely linear algebra point of view to the image reconstruction process, the noise floor can be understood with the help of Figure 8. A true brightness temperature distribution $\mathrm{x}_{\mathrm{T}}$ in $\mathrm{R}^{\mathrm{n}}$ space (with $\mathrm{n}$ being large) maps into $\mathrm{y}$ in the visibility domain in $\mathrm{R}^{\mathrm{m}}$ ( $\mathrm{m}$ also being large). However only a limited set of visibilities are actually measured, which in turn defines a fundamental hexagonal region in the physical space, smaller than the unity circle. Therefore only a visibility vector hat $\{y\}$ projected onto the column space of the -assumed perfectly known- G matrix is available for inversion. Using the pseudo-inverse matrix of $G, G^{+}$, a least squares solution hat $\{x\}$ is found, which belongs to the row space of the $G$ matrix, at some unavoidable distance from the true brightness temperature distribution $\mathrm{x}_{\mathrm{T}}$, this distance defining the noise floor. The noise floor is therefore the component of the true brightness temperature vector in the direction of the null space of the G matrix, and is therefore proportional to the brightness temperature of the scene.

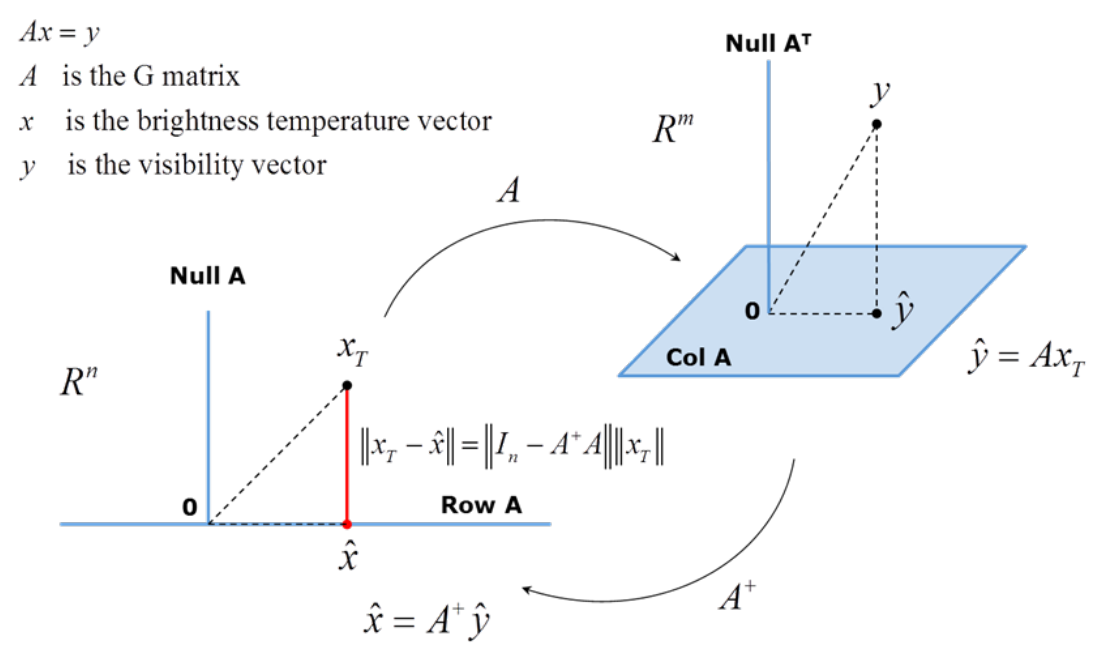

Figure 8: Noise floor definition

The following methods have been attempted, at Level-1 data processing, to mitigate the noise floor:

a) Amplitude Mask 


\section{REPLACE THIS LINE WITH YOUR PAPER IDENTIFICATION NUMBER (DOUBLE-CLICK}

HERE TO EDIT) <

276 This technique is based on assuming that the spatial ripple can be mitigated by the use of a 277 multiplicative mask built over uniform targets like the Southern Pacific Ocean or Antarctica (Lin et al., 2011, 2012)(Torres et al., 2012).

b) Floor Error Mask (FEM)

A difference brightness temperature map is built between the measurements and what an ideal instrument would reconstruct. Such map is produced over a full year, and split into a scene dependent and a scene independent components. The latter is used to correct SMOS images for the scene independent spatial ripple (Anterrieu et al., 2015).

c) Pre-Distorted G-matrix (PDG)

Using the Binomial Inverse Theorem the G-matrix is pre-distorted a priori in order to reduce the error contribution due to antenna pattern differences (Díez-García et al., 2014).

d) Average Pattern Reconstruction (APR)

The visibilities are decomposed in two components, one corresponding to an instrument with the visibilities and an image reconstruction using an instrument with identical antenna and receivers is performed.

e) Initial guess based techniques

These methods are based on the second result stated in the section devoted to the spatial ripple, i.e. that the scene content outside the alias free field of view has an impact on the recovered scene inside it. According to this result, it is desirable to reduce the brightness temperature content outside the alias free field of view, which can be achieved if a first guess of the scene is subtracted prior to the image reconstruction. The set of methods implementing this technique is referred to as "Gibbs" methods, as they try to reduce the contrast within the scene, hence the Gibbs ringing, and by extension, the spatial ripple (Camps et al., 2008)(Khazaal et al., 2009) (Corbella et al., 2014). There are different levels of Gibbs techniques with increased implementation complexity: Gibbs-0 assigns 


\section{REPLACE THIS LINE WITH YOUR PAPER IDENTIFICATION NUMBER (DOUBLE-CLICK}

HERE TO EDIT) <

a single value to the whole unity circle; Gibbs-1 differentiates between the sky and the Earth disk, giving a single value of brightness temperature to the whole Earth disk, and another one to the sky; Gibbs-2 introduces, in addition, a differentiation within the Earth disk between land and ocean masses, assigning a constant but different value to each part; an option of Gibbs-2 includes a Fresnel variation over incidence angle over the ocean, instead of using a single value; in Gibbs-3 the first guess is obtained from the long record of measured brightness temperatures as opposed to the use of models, as done in the previous cases.

The Gibbs-n techniques have been, among all, the most successful ones in reducing the spatial ripple.

The success of the Gibbs-n techniques depends on the amplitude of any residual calibration errors.

311 Nonetheless, because of the existence of the noise floor, it is unlikely that the current level of spatial 312 ripple be significantly reduced in the future. The current Level-1 processor implements a Gibbs-1 313 technique, the remaining spatial ripple being removed at Level-2 through the Ocean Target

314 Transformation (Gourion et al., 2013), to enable the retrieval of Sea Surface Salinity.

\subsubsection{Correction of Sun and RFI Tails}

Given the element spacing in SMOS, the aliases of the Sun are unavoidable. The mitigation of the side

319 lobes joining the real Sun and its aliases, visible in the left panel of Figure 9 with peak amplitudes of 320 the order of $-18 \mathrm{~dB}$, should be achievable through the application of a stronger apodization window in 321 the spatial frequency domain than the nominal Blackman window (Camps, 1996) (Anterrieu et al., 322 2002). When a Circular window with much increased tapering than the nominal Blackman is applied to 323 the spatial frequencies, the simulated response of the center panel should be obtained. Instead, the 324 measurements, shown in the right panel, reveal that the side lobe level remains roughly at the same 325 level (Torres et al., 2014). This puzzling result is a consequence, again, of the combination of the alias 326 condition and the dissimilarities across the antenna patterns, as was the spatial ripple. In other words, 


\section{REPLACE THIS LINE WITH YOUR PAPER IDENTIFICATION NUMBER (DOUBLE-CLICK HERE TO EDIT) <}

327 the noise floor sets a limit in the control that can be reached on the side lobes through windowing in the 328 spatial frequency domain.
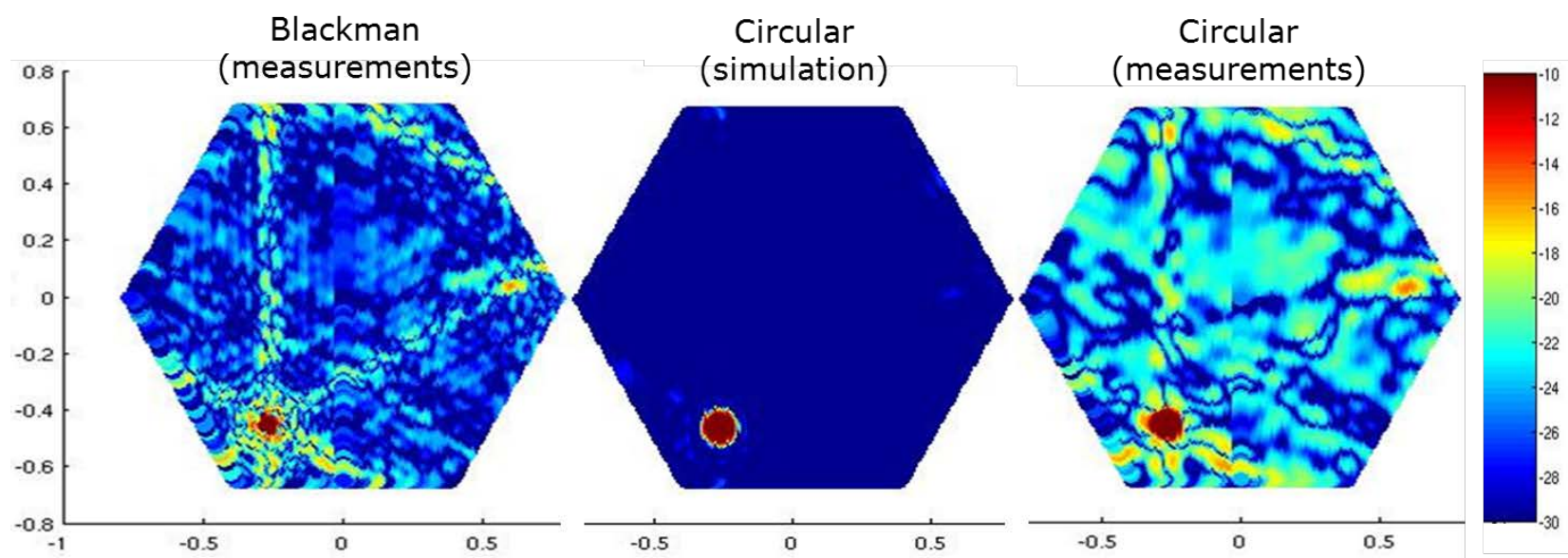

Figure 9: Measured Sun response with standard Blackman window (left), expected image with a stronger circular apodization window (center) and the corresponding measurements (right). Colour scale gives the brightness temperature in logarithmic scale (dB-K).

The following other techniques have been attempted to remove the Sun alias and its side lobes from the

SMOS images:

a) Use of a measured Sun response

During one orbit of the $9^{\text {th }}, 10^{\text {th }}, 11^{\text {th }}$ and $13^{\text {th }}$ of January 2013 , SMOS was pointed towards the galactic pole at a time when the Sun elevation was $21.3^{\circ}, 17.1^{\circ}, 10.1^{\circ}$ and $21.6^{\circ}$, respectively, above the antenna plane. Four Sun responses were acquired over the uniform low cold sky background. These responses were later translated in position and scaled in amplitude, to correct for the Sun during measurements in nominal pointing. This method is still under research

b) Use of an estimation of the Sun response

From an image contaminated by the Sun, the position and amplitude of the Sun are estimated, an artificial response is built based on them, and then subtracted from the original image (Camps et al., 2004). In this correction technique the most critical parameter is the accuracy in the localization of the Sun, which depends on the number of solar spots (Chiuderi Drago et al. 
REPLACE THIS LINE WITH YOUR PAPER IDENTIFICATION NUMBER (DOUBLE-CLICK

HERE TO EDIT) <

1977). An iterative process to estimate the position and brightness temperature of the Sun has shown promising results, but the increase in computational time is critical and is still under evaluation. A simpler method has been implemented in the Level-1 data processor of SMOS, with limited improvement, and has been complemented by flagging. Figure 10 shows an example of the Sun correction.
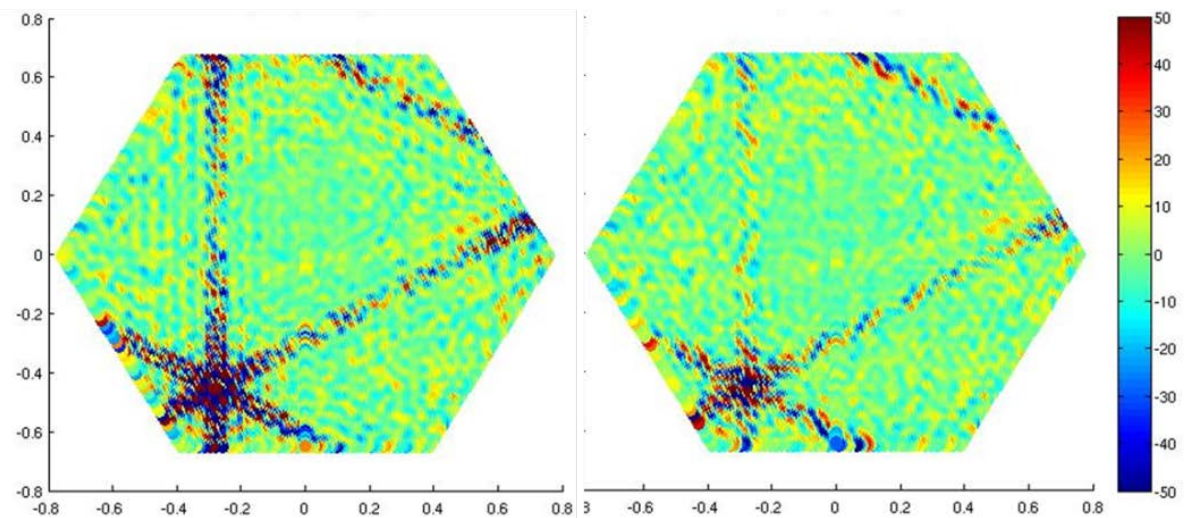

Figure 10: SMOS image of the Sun in cold sky pointing mode before (left) and after (right) Sun correction. Colour scale is in Kelvin.

In principle, the methods for the correction of the Sun alias and its tails can also be applied to remove

RFI sources effects. However, the population of RFI sources is irregular, clustering in some regions of the Earth, with several interferors appearing at the same time inside the field of view of SMOS. In this situation a correction for the RFI sources of the type described for the Sun is difficult. Nonetheless, techniques to better detect, flag and correct for RFI sources keep being developed and assessed (Khazaal et al., 2014). In parallel, a technique called the Nodal Sampling (González-Gambau et al., 2015) has been proposed to image RFI polluted areas and is under assessment, showing some promising results.

\subsubsection{Correction of Land-Sea Contamination}

During the investigation of the land-sea contamination error it has become clear that a mismatch between the amplitude of the visibility at the origin $\mathrm{V}(0,0)$ and the rest of the samples, $\mathrm{V}(\mathrm{k}, \mathrm{j})$, generates this kind of degradation, as shown in the left plot of Figure 5. Empirically it has been proven that 
REPLACE THIS LINE WITH YOUR PAPER IDENTIFICATION NUMBER (DOUBLE-CLICK HERE TO EDIT) <

373 affecting the correlator efficiency coefficients $G_{k j}$ of the visibility samples $V(k, j)$ outside the origin by a 374 factor near 0.98 removes significantly the land-sea contamination. The right plot of Figure 5 shows the 375 improvement when this correction is applied. The warm brightness temperature halos surrounding the 376 continents have mostly disappeared. The possibility of correcting the correlation efficiency coefficients $377 \mathrm{G}_{\mathrm{kj}}$ has been implemented in the latest version of the SMOS Level-1 processor (V700) and will be 378 subject of validation before it is accepted to enter into operation.

379 It is worth mentioning that a parallel empirical correction of the land-sea contamination is being 380 prepared at Level-2 based on a mask built with the 5 year long record of SMOS data (SMOS Level-2 381 Ocean Salinity Team, 2015).

\subsubsection{Correction of Seasonal and Orbital Variations}

The seasonal and orbital variations are observed in the right plots of Figures 6 and 7 respectively as warm anomalies around October and in the eclipse season every year. The current strategy to correct

387 for these fluctuations is to simplify the calibration approach of the instrument as much as possible by 388 using the All-LICEF mode (Torres et al., 2006) explained below and then attempt new corrections to 389 mitigate them.

\section{IN FLIGHT CALIBRATION PLAN}

\section{$392 \quad$ 4.1 The Corbella Equation}

393 The Corbella equation, introduced in 2003 (Corbella et al., 2004a), involves a fundamental 394 modification to the formulation of interferometry, as used in radio-astronomy, that is necessary to 395 describe the way an aperture synthesis radiometer of the type of MIRAS works. The calibration of 396 SMOS is based on the Corbella equation, and hence, one of the first and most important tasks 397 undertaken in the frame of the calibration of the instrument was its verification (Martín-Porqueras et 398 al., 2010). Such exercise would ideally involve the imaging of two perfectly uniform black body targets 


\section{REPLACE THIS LINE WITH YOUR PAPER IDENTIFICATION NUMBER (DOUBLE-CLICK HERE TO EDIT) <}

399 at two different physical temperatures. Since the Cold Sky near the galactic pole is the only reasonable 400 realization to such uniform target, the validation of the Corbella equation focused on the Cold Sky. 401 With the help of a simulator, two sets of visibility samples of the Cold Sky were produced using the 402 radio astronomy and the Corbella equations. Then, to improve contrast, the visibility samples at the 403 origin were set to zero, and a simple Fourier Transform was applied to provide the images of the 404 modified brightness temperature of the Cold Sky in each case. The image obtained using the radio 405 astronomy formulation is shown in the left panel of Figure 11, while the one resulting from the use of 406 the Corbella equation is in the right panel. These images were then compared to the one measured by 407 SMOS, shown in the center panel of Figure 11, which was generated in the same way, i.e. through a 408 Fourier Transform of the measured visibility samples setting the one at the origin to zero. The image 409 using the Corbella equation is very similar to that measured by SMOS, while the Cold Sky retrieved 410 with the radio astronomy equation does not capture any of the features present in the measurements.

411 This test validated the Corbella equation.

SMOS

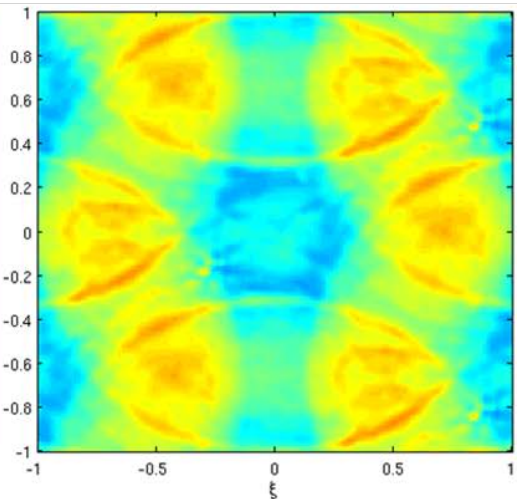

Corbella

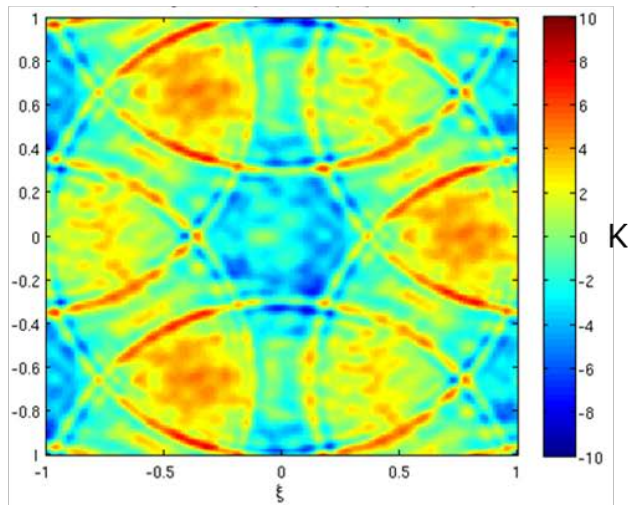

Figure 11: Expected modified brightness temperature of the Cold Sky using the radio astronomy (left) and the Corbella (right) equation; center is the SMOS measurement (note: the visibility sample at the origin has been set to zero to improve contrast)

\subsection{Routine In-orbit Calibration Plan}

The routine in-orbit calibration plan was established at the end of the Commissioning Phase, in May 2010. With the experience of the first year of the operational phase (Oliva et al., 2013), weekly Short Calibrations while flying over Antarctica were added as from March 2011 to track the temporal 
REPLACE THIS LINE WITH YOUR PAPER IDENTIFICATION NUMBER (DOUBLE-CLICK HERE TO EDIT) <

421 variation of the voltage offset of the receivers, leading to the calibration plan shown in Figure 12, 422 which is the one currently used in SMOS.

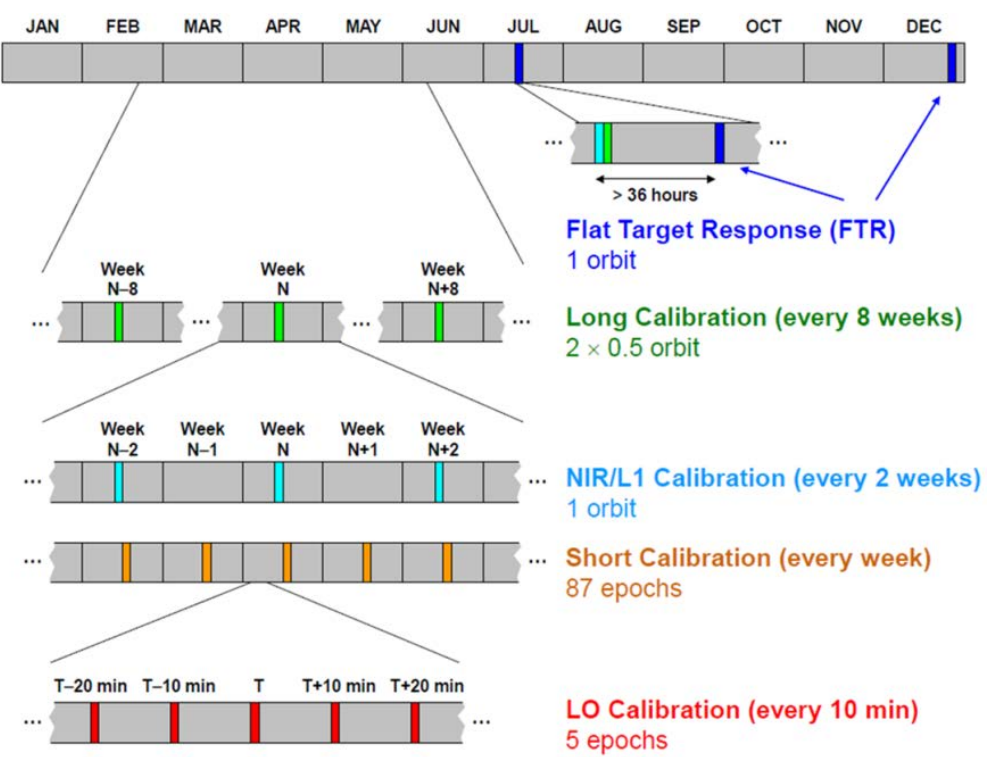

Figure 12: SMOS routine in-orbit calibration plan

\subsection{In-flight Calibration Improvements}

429 Two improvements have been made based on the in-flight experience over these 5 years: the "warm" 430 external calibrations and the addition of an RFI check to validate the external calibrations.

\subsubsection{Warm Calibrations}

Detailed analysis of the external calibrations revealed that a few LICEF receivers of MIRAS exhibited small and smooth unexpected jumps in their PMS (Power Monitoring System) detector voltages. These jumps seemed to correlate well with the skin temperature of the antenna, happening more frequently for colder skin temperatures, and appeared to be reversible in the sense that, for warmer skin temperatures, the usual values were again obtained. To illustrate this, refer to the left panel in Figure 13, which spans one full orbit flown pointing zenith during the Commissioning Phase, including the transitions from and to Earth pointing at the beginning and end of the plot, respectively. The 3 cyan curves provide the skin temperature of the antenna measured by 3 thermistors (named Tp7). The black and green lines give, respectively, the elevation of the Sun over the antenna plane and its azimuth, in decadegrees as 
REPLACE THIS LINE WITH YOUR PAPER IDENTIFICATION NUMBER (DOUBLE-CLICK HERE TO EDIT) <

442 read from the scale on the right. The Sun elevation is negative (the Sun is behind the antenna) except 443 for a portion in the right half of the plot, where it reaches an elevation of about $30^{\circ}$. As soon as the Sun 444 appears in front of the antenna the skin temperature (cyan lines) increases from near $0^{\circ} \mathrm{C}$ till some $44520^{\circ} \mathrm{C}$, to return back to just $1^{\circ} \mathrm{C}$ or $2^{\circ} \mathrm{C}$ as the Sun sets behind the antenna horizon. The dark blue and 446 dark red crosses correspond to the detected voltages in the vertical and horizontal polarizations. The 447 vertical polarization shows some fluctuations. The first anomaly appears towards the left of the plot 448 when the physical temperature drops below some $10^{\circ} \mathrm{C}$. At this moment the detected voltage jumps up 449 a few millivolts, which is unexpected because as the instrument is pointed towards cold sky the 450 detected voltage is expected to constantly decrease towards a minimum level. The anomalous higher 451 value is maintained until the temperature rises again above some $12^{\circ} \mathrm{C}$. Then several fluctuations 452 happen centered around the maximum of temperature in the right half of the plot, showing a high 453 degree of symmetry and correlation with the temperature evolution. The detected voltage attains a right 454 value only in the center of these fluctuations, coinciding with the warmest temperature interval around 455 the peak. It is plausible that these fluctuations could be related to a change in the electrical phase of 456 some Teflon pieces of the antenna at some physical temperature range. Another example is given in the 457 right panel of Figure 13, which corresponds to another of the affected receivers, this time during a 458 typical external calibration manoeuvre: as the antenna cools down and its temperature reaches about $4592{ }^{\circ} \mathrm{C}$, the detected voltages at the two polarizations experience jumps of 10 and $40 \mathrm{mV}$ about. The 460 purple line in the right panel is the estimated physical temperature at which the Teflon parts of the 461 antenna could be. To avoid these voltage jumps, the external calibration manoeuvres are planned, since 462 October 2014, at a modified time to have the Sun at some positive elevation angle over the antenna 463 plane. The Sun illumination on the antenna keeps it warm, avoiding the skin temperature to fall too low 464 and the PMS detector voltage fluctuations. The positive Sun elevation is however kept below a limit of $46510^{\circ}$ to ensure that its presence does not degrade the external calibration acquisitions. Careful checks were carried out to detect any effect from the direct signal of the Sun that could compromise the quality 


\section{REPLACE THIS LINE WITH YOUR PAPER IDENTIFICATION NUMBER (DOUBLE-CLICK HERE TO EDIT) <}

467 of the external calibration. In addition the warm calibrations perform the external calibration with the 468 antenna at a more similar temperature to the measurement mode, which is desirable, and reduce thermal 469 excursion on the antenna, improving reliability. Warm calibrations can be planned any time along the 470 year except around the equinoxes, when the Sun elevation is just too low, in which case, the usual cold 471 calibrations are performed instead.
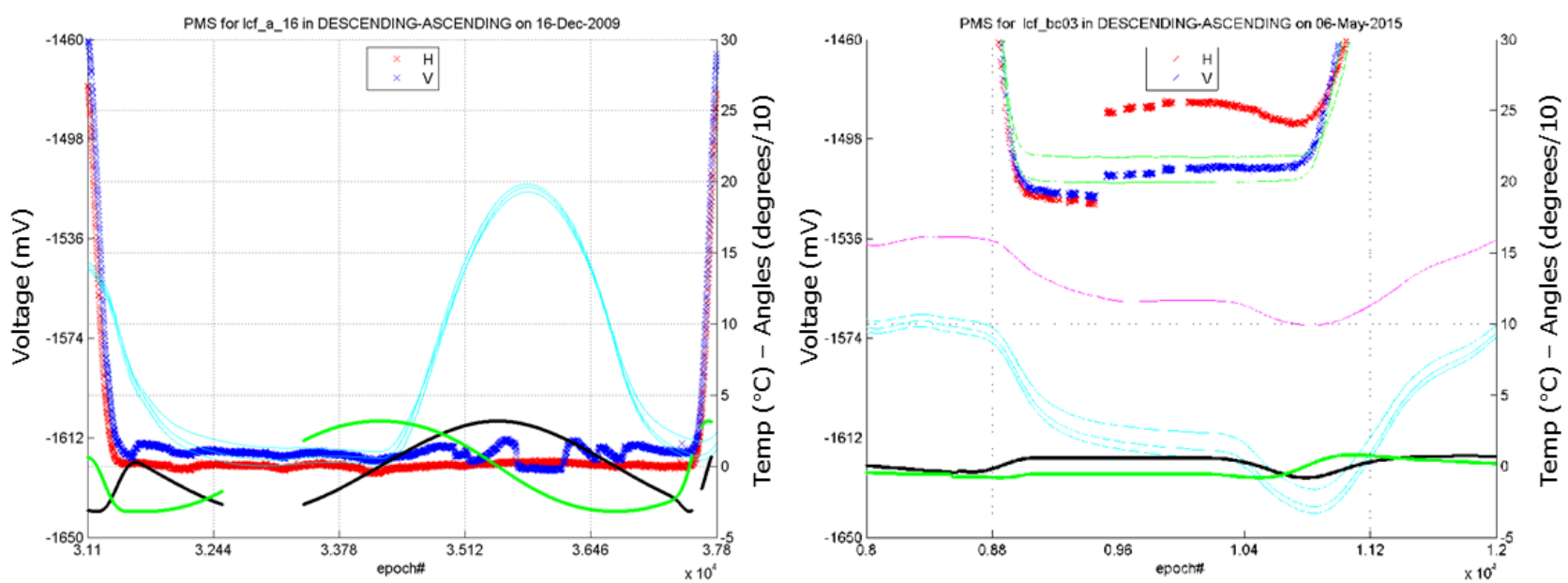

Figure 13: Example of PMS detector voltage fluctuations in a zenith pointing orbit (left) and during a typical external calibration (right) that led to the introduction of the external 'warm' calibrations

\subsubsection{RFI Check in Validation of External Calibrations}

External calibrations, where SMOS is pointed towards the Cold Sky, are executed only over the Pacific Ocean to avoid picking up signals from strong RFI transmitters on ground through the back lobes of the antennas. However, in one instance, an external calibration carried out 3 June 2015 in the NorthEastern Pacific Ocean, near Alaska, appeared contaminated by some ground interference. This caused some disturbance in the data production chain as the calibration file had been ingested before the problem was discovered. To avoid this, since then, every external calibration (these are performed once every 2 weeks) is manually checked for RFI degradation before being accepted for use in the Level-1 data processor. An automatic procedure is being built up to replace the manual check. 
REPLACE THIS LINE WITH YOUR PAPER IDENTIFICATION NUMBER (DOUBLE-CLICK

HERE TO EDIT) <

\section{$488 \quad 5 \quad$ IN FLIGHT INSTRUMENT MONITORING}

490 The values of some of the key instrument parameters which are monitored or calibrated in flight are 491 presented next.

\section{$492 \quad 5.1 \quad$ Physical Temperature}

\section{$493 \quad$ 5.1.1 Skin Antenna Temperature}

MIRAS carries a thermistor (labeled Tp7) inside the head of the central screw of the antenna of the 3

496 Noise Injection Radiometers whose readings are representative of the physical skin temperature of any 497 of the antennas (Rubiales et al., 2015). This temperature is important because it affects the amount of 498 noise emitted by the front end equivalent resistor. It also provides an indication on how different the 499 thermal conditions of the antenna are between an external calibration and the nominal measurement 500 mode.

501 The evolution of the temperature readings from the $3 \mathrm{Tp} 7$ thermistors is shown in Figure 14 . The skin 502 temperature goes through its largest excursion (from about $6^{\circ} \mathrm{C}$ to $28^{\circ} \mathrm{C}$ ) during every boreal winter 503 solstice, when the Sun reaches maximum elevation above the antenna plane (around $31^{\circ}$ ) and is 504 eclipsed by the Earth. There is a second period of large thermal excursion (from $8^{\circ} \mathrm{C}$ to $18^{\circ} \mathrm{C}$ 505 approximately) around every boreal summer solstice where the Sun elevation reaches up to $15^{\circ}$ 506 elevation above the antenna horizon. During the equinoxes the temperature excursion is the smallest 507 (between $5^{\circ} \mathrm{C}$ to $12^{\circ} \mathrm{C}$ ) and the lowest skin temperatures are recorded, except for the external 508 calibration events. The latter correspond to the individual spikes that drop below $0^{\circ} \mathrm{C}$ in Figure 14 . The 509 Tp7 temperatures went through an initial cooling transient, clearly observed during the first half of 5102010 , to then flatten out into a very small long term residual cooling trend. 


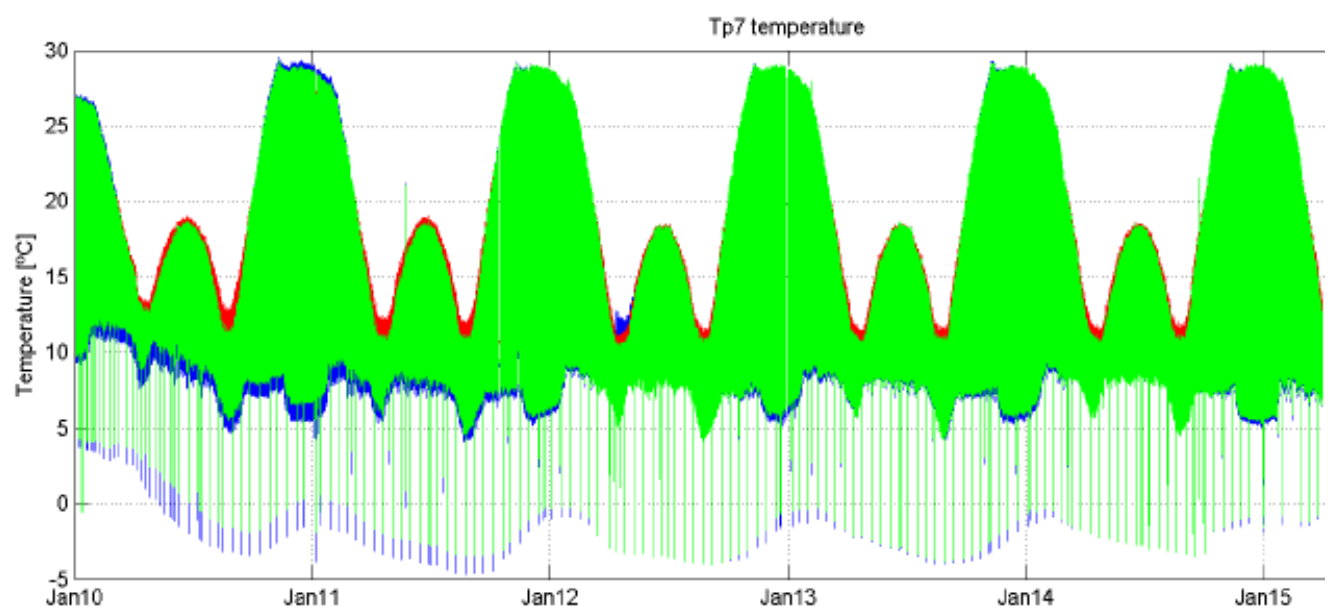

Figure 14: Evolution of the skin antenna temperature measured by the $3 \mathrm{Tp} 7$ thermistors

\subsubsection{Inner Receiver Temperature}

Every one of the 72 LICEFF receivers of MIRAS has a thermistor (labelled Tp6) next to an internal matched load in the front-end electronics used as warm point in the amplitude calibration. This thermistor senses the inner temperature of the receiver. The average value of Tp6 across all LICEF receivers is shown in Figure 15. The physical temperature of the receivers is seen to be quite stable along the mission, centered around $22^{\circ} \mathrm{C}$ with a global peak to peak variation of about $1{ }^{\circ} \mathrm{C}$. As for $\mathrm{Tp} 7$, the Tp6 readings present larger excursions during the solstices, and narrower variation around the equinoxes, where its lowest values are attained.

\subsection{Receiver Parameters}

\subsubsection{Antenna Losses}

The antenna has two distinct loss components: one due to the radiating resonant cavity, and the other due to the intermediate layer circuit that combines the signal from the pair of probes of each polarization. The first component is tiny and difficult to measure on ground. It was estimated to be of about $\mathrm{L} 1=0.05 \mathrm{~dB}$, by calculations based on the geometry and materials of the antenna design. On the other hand, the losses of the intermediate layer circuit, of about L2 $=0.25 \mathrm{~dB}$, was measured on ground. The total antenna losses are then expected to be around $0.30 \mathrm{~dB}$. During the in orbit calibration, the 
REPLACE THIS LINE WITH YOUR PAPER IDENTIFICATION NUMBER (DOUBLE-CLICK HERE TO EDIT) <

535 antenna loss are directly measured using the Cold Sky and the internal matched load (Corbella et al., 536 2012). The average value across all LICEF receivers for each polarization is shown in Figure 16. The in-flight measured antenna losses are about $0.17 \mathrm{~dB}$ larger than their pre-launch estimated value. It is worth noticing the rapid evolution exhibited during the first 6 months of the mission, as well as the seasonal fluctuations, the latter being partly driven by the PMS detector voltage fluctuations described earlier. The antenna losses present a different evolution after October 2014, reflecting the introduction of the warm external calibrations to avoid the mentioned PMS fluctuations.

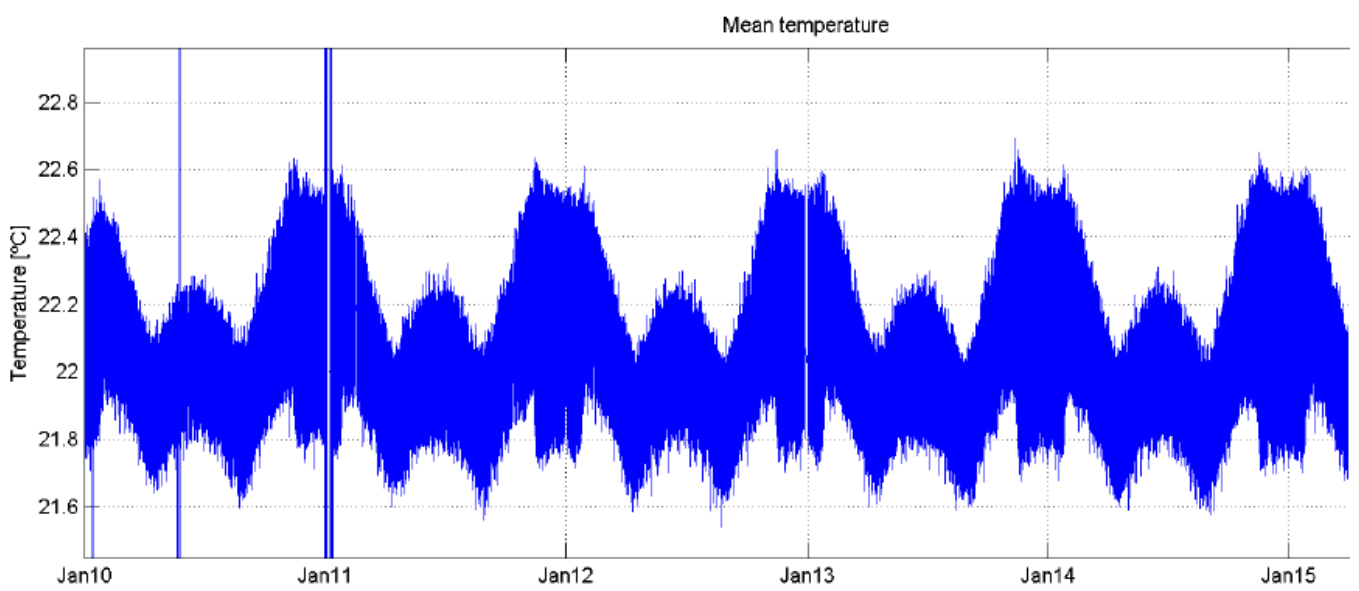

Figure 15: Average inner LICEF receiver temperature Tp6. The spikes in early Jan’10, May'10 and Jan'11 are due to 3 anomalies occurred in the instrument.

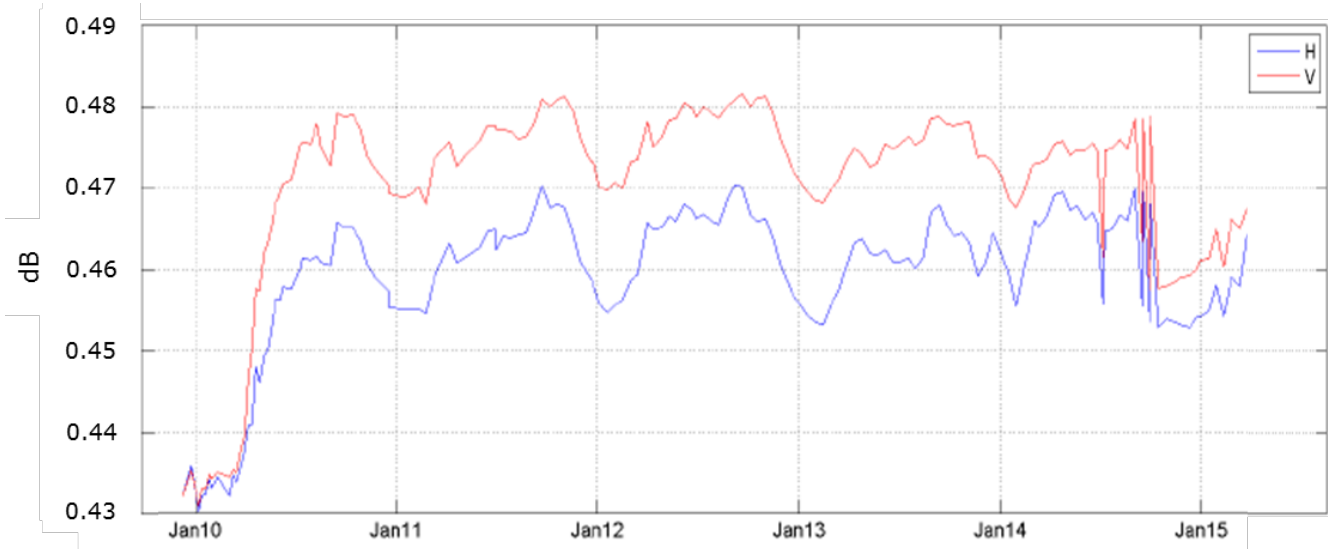

Figure 16: Evolution of the antenna losses as measured in flight

\subsubsection{Receiver Detector Gains}

The average detector gain across all LICEF receivers is presented in Figure 17 for each polarization.

The absolute gain is shown in the left panel. Similar features to those found in the evolution of the 


\section{REPLACE THIS LINE WITH YOUR PAPER IDENTIFICATION NUMBER (DOUBLE-CLICK HERE TO EDIT) <}

555 antenna losses are repeated here: an initial rapid transient followed by seasonal variations. In addition 556 the receiver gains seem to be undergoing an exponential decay which, according to the relative gain 557 variation shown in the right panel, has accumulated a total decrease of about $1.5 \%$. The reason for this decay is unknown, but could be caused by the overall thermal stabilization over mission life time.

\subsubsection{Receiver Detector Voltage Offets}

The average voltage offset across all LICEF receivers is shown in Figure 18. The behavior is somewhat erratic, without any clear trend, with rapid fluctuations that led, in March 2011, to the introduction of weekly short calibrations as from March 2011 to track them. The voltage offset is therefore well followed with a weekly refresh rate and calibrated out.
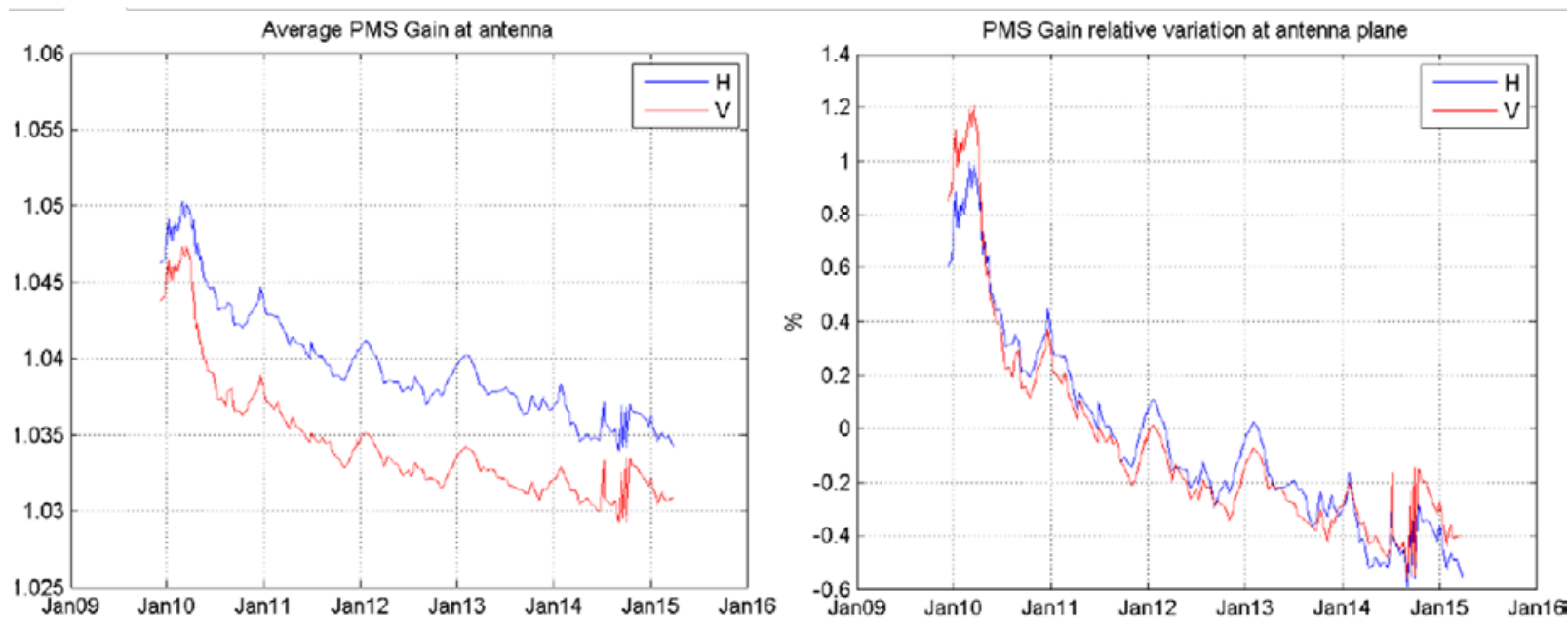

Figure 17: Evolution of the end-to-end average receiver gain in $\mathrm{mV} / \mathrm{K}$ (left) and in percentage variation taking June 2011 as reference value (right)

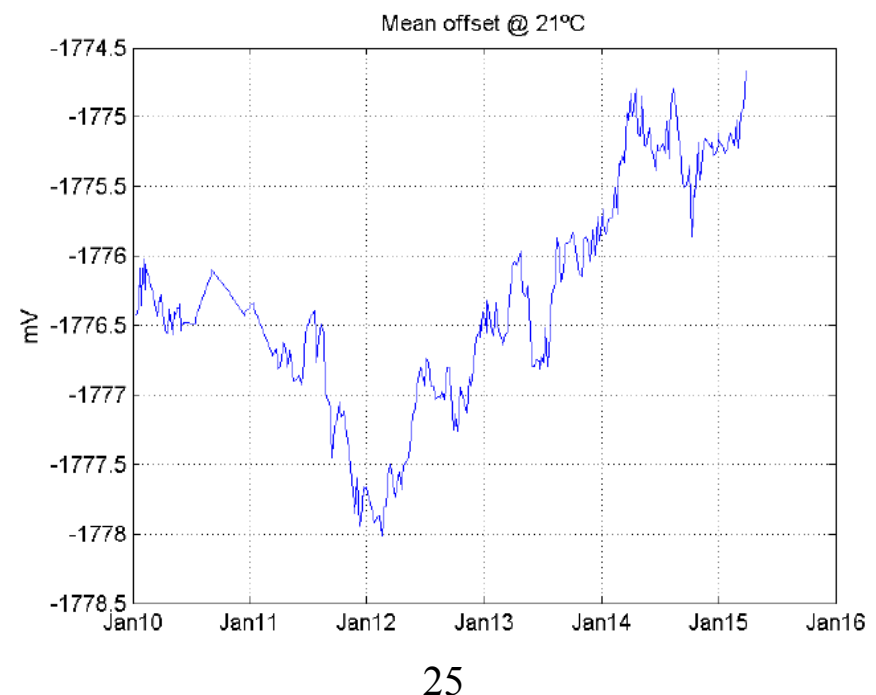


REPLACE THIS LINE WITH YOUR PAPER IDENTIFICATION NUMBER (DOUBLE-CLICK HERE TO EDIT) <

573

574 575

576

577

578

579

580

581

582

583

584

585

Figure 18: Evolution of the average detector offset voltage (in $\mathrm{mV}$ ) across all LICEF receivers

\subsection{Baseline Parameters}

\subsubsection{Relative Phase across Receivers}

The relative phase across receivers is measured once every 10 minutes through the injection of a burst of correlated noise into all LICEF receivers. Phases between receivers sitting in different arm segments, hence fed by physically different local oscillators, present the strongest temporal variations. In addition some of the 12 phase-lock-loop circuits available on board lose lock from time to time for reasons still unknown, but probably due to temperature (in total 9 unlocks per year, on average), generating phase jumps. As an example, the phase between one receiver in the hub and other receiver in the first segment of one of the arms of MIRAS is shown by the blue line in the left panel of Figure 19. After correcting for any unlocks (blue spikes), the corrected phase in red is obtained. The fluctuations of the corrected phase are correlated with the physical temperature, which causes slight differential changes in the two local oscillators involved in the particular baseline.

On the other hand, the relative phase across receivers fed by the same local oscillator, i.e. sitting in the same arm segment, is rather stable, and are not affected by the unlocks. The right panel of Figure 19 brings the evolution of the relative phase across the 6 receivers of the third segment of one of the arms, shifted vertically only for presentation purposes. The peak to peak phase fluctuations are below $1^{\circ}$ across the entire mission except for a few jumps that can be attributed to one specific receiver, probably caused by thermal expansion in one cable connection, but for which there is no proven explanation. 


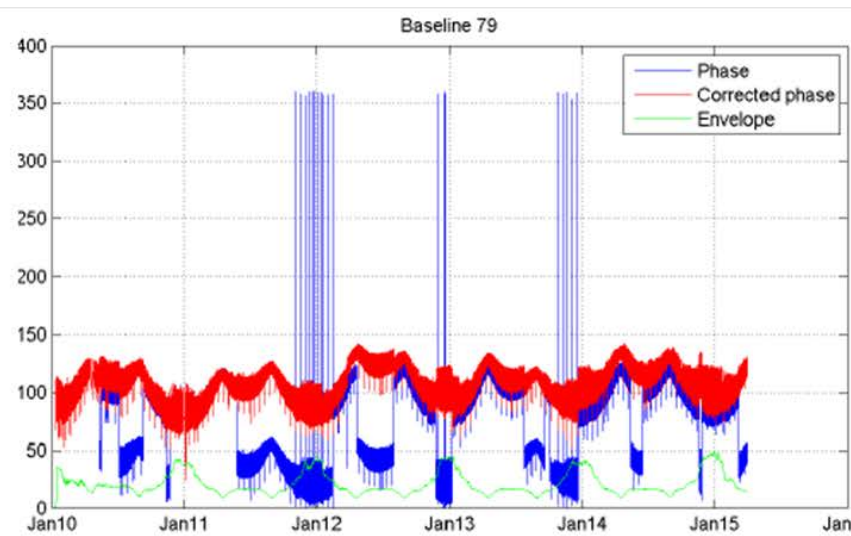

\subsubsection{Correlator Amplitude Coefficients} significant temporal trend.

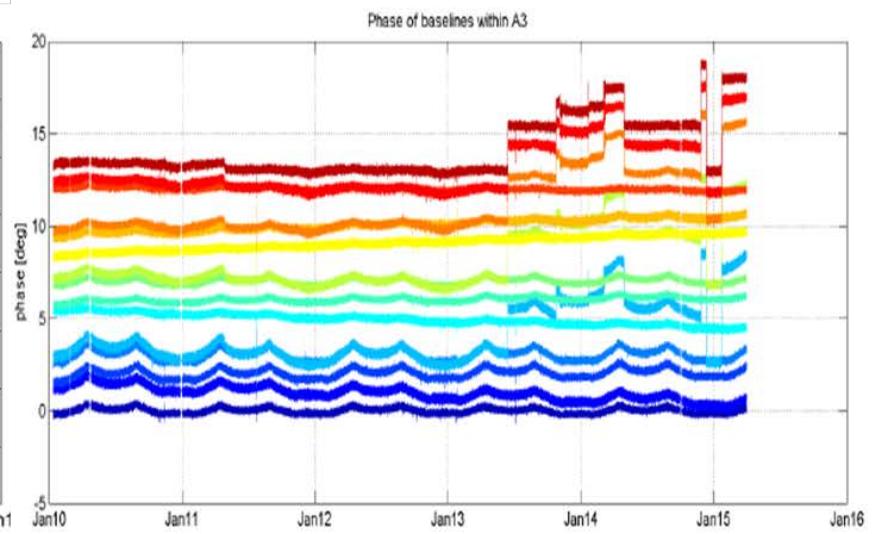

Figure 19: Relative phase between 2 LICEF receivers which do not share the same local oscillator (left) - spikes correspond to $360^{\circ}$ wrappings - and across each of the 15 possible pairs from the set of 6 LICEF receivers of one arm segment connected to the same local oscillator (right)

The average value of all the 2556 efficiency coefficients $G_{k j}$ of the correlator for each baseline formed by receivers $\mathrm{k}$ and $\mathrm{j}$ is shown in Figure 20 . These $\mathrm{G}_{\mathrm{kj}}$ coefficients represent the value of the so-called Fringe-Washing Function (FWF in short) at zero delay. They provide the correlation losses due to the on-board calibration network and the differences in the frequency responses of the receivers. The $G_{k j}$ coefficients are used to denormalize the value of the raw correlations obtained from the 1-bit sampled signals. In theory the $G_{k j}$ cannot be larger than 1 , but because they are obtained through a combination of measurements of correlated noise injection from common Noise Sources (NS) and closure equations, some of them reach values slightly above 1 . The $G_{k j}$ coefficients are very stable along the mission. The average of all $\mathrm{G}_{\mathrm{kj}}$ is about 0.99 , without any 


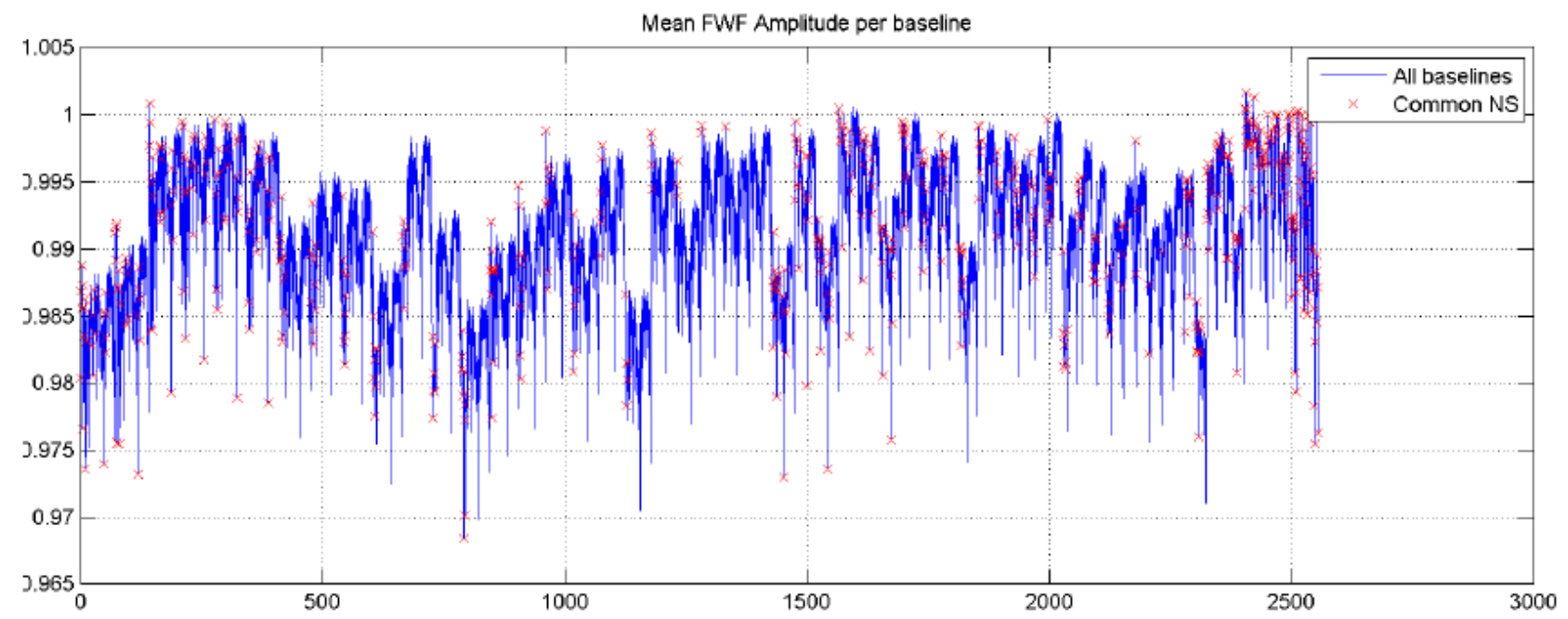

Figure 20: Average value of the $\mathrm{G}_{\mathrm{kj}}$ correlator efficiency coefficients

\subsection{Noise Injection Radiometer Parameters}

The 3 Noise Injection Radiometers (NIR) embarked on SMOS are the reference radiometers of MIRAS (Colliander et al., 2007). They serve several purposes: (a) they measure the visibility sample at the origin; (b) they provide the reference antenna temperature to de-normalize the visibility samples obtained through 1-bit correlations; (c) they measure the amplitude of the noise diodes of the on-board Calibration System (Lemmetyinen et al., 2007); and (d) they are used as an additional receiver to generate visibility samples. A summary of their in orbit performance is presented next.

\subsubsection{NIR Long and Short Term Stability}

The NIR long term stability is illustrated in the left panel of Figure 21, which gives the error in each of the two polarizations against the Cold Sky for all external calibrations manoeuvres, performed once every 2 weeks, when a single calibration point, at the beginning of the Operational Phase (June 2010) is used. As it is shown, after the transient of the first 6 months of the mission (Kainulainen et al., 2010, 2012), the stability of the NIR units is remarkable: even when being calibrated only once in 4 years, the long term drift is only of about $-0.1 \mathrm{~K} /$ year. The right panel of Figure 21 shows the short term stability of the NIR units by providing the Cold Sky error just before the NIR parameters are refreshed at every external calibration. The accumulated error over 2 weeks is within $\pm 0.4 \mathrm{~K}$. The bi-weekly external 
REPLACE THIS LINE WITH YOUR PAPER IDENTIFICATION NUMBER (DOUBLE-CLICK HERE TO EDIT) <

637 calibrations remove completely any long term drift. The annual behavior of the NIR error, that is 638 clearly seen in Figure 21 (left) is investigated a lot during the mission. Trials have been made to 639 relate the drift to internal instrument properties like physical temperatures, and to external 640 conditions, like Sun position or other components that contribute to the total antenna 641 temperature. Contributions of such external sources are reviewed e.g. in (Colliander, 2015), in 642 which forward geophysical model simulations were used to simulate NIR antenna temperature measurements.

644 The observed $0.13 \mathrm{~K}$ standard deviation error of the sky measurements in Figure 21 scales down to about $0.1 \mathrm{~K}$ error in measurement pointing mode (Kainulainen et al, 2012), a smaller error than the $0.2 \mathrm{~K}$ radiometric resolution of the NIR units, and thus, not affecting the retrieval of the 647 geophysical parameters.
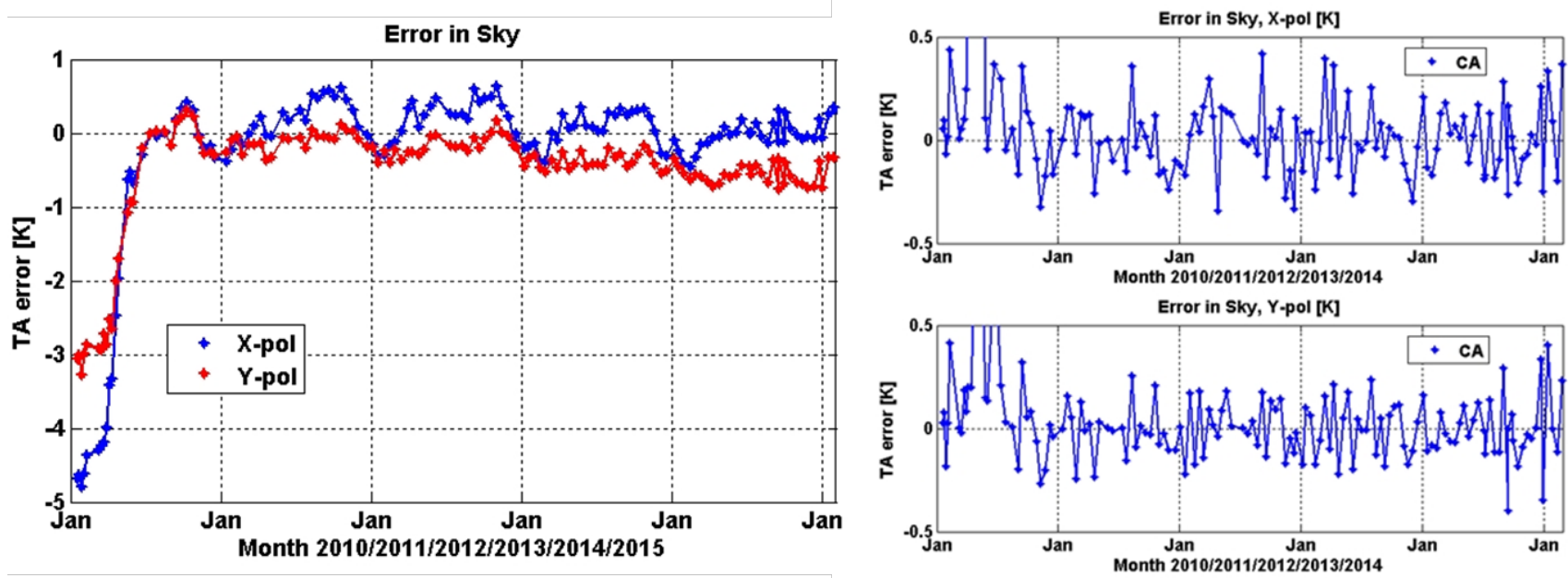

Figure 21: NIR-CA stability when calibrated only once (left) or every 2 weeks (right)

\subsubsection{Noise Injection Temperatures}

NIR uses some internal noise diodes to operate. The stability of these diodes and their injection circuits are critical to establish the performance of the NIR units and the whole MIRAS instrument. Two levels of noise injection temperatures are used, a low one $\mathrm{T}_{\mathrm{na}}$ to measure the antenna temperature, and a high one, $\mathrm{T}_{\mathrm{nr}}$ to measure the diodes of the on-board Calibration System. Figure 22 shows the 5 year evolution of $\mathrm{T}_{\mathrm{na}}$ and $\mathrm{T}_{\mathrm{nr}}$ in the left and right panels respectively, for the horizontal polarization (similar 
REPLACE THIS LINE WITH YOUR PAPER IDENTIFICATION NUMBER (DOUBLE-CLICK HERE TO EDIT) <

659 results are obtained in vertical polarization). Besides the initial transient, the variations over 5 years are 660 within $0.2 \%$ peak to peak for the most stable unit, NIR-CA.
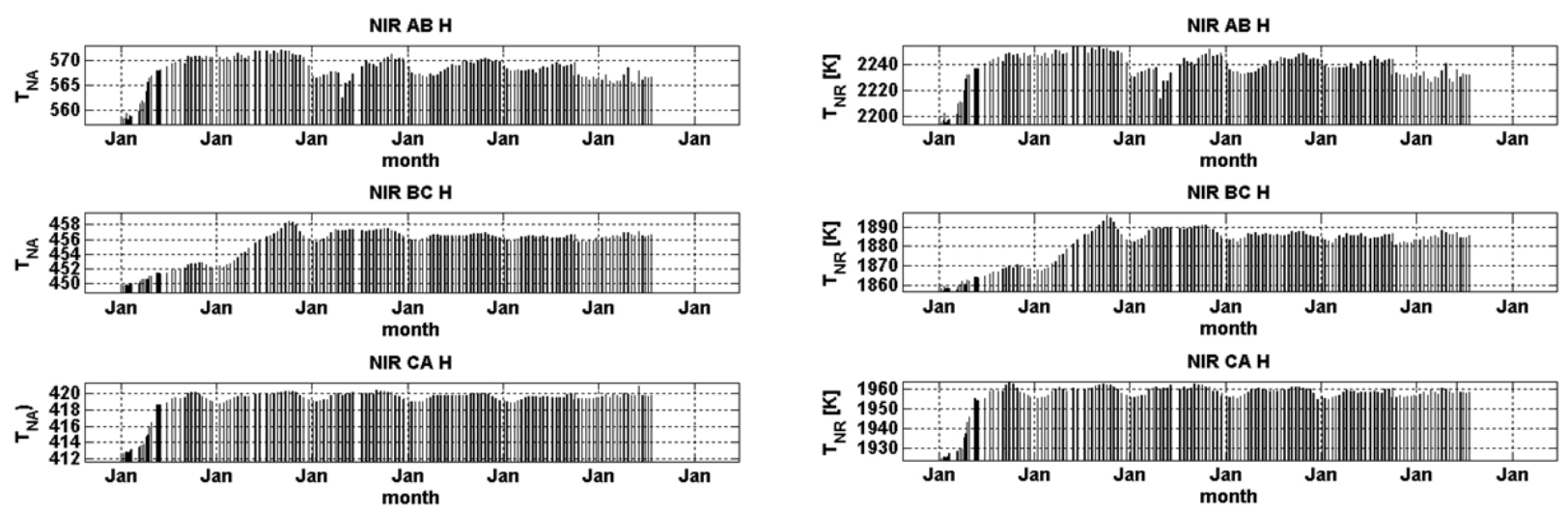

Figure 22: Temporal evolution of the noise injection temperatures of the NIR units

667 samples into calibrated brightness temperature records. The first version used during the 668 Commissioning Phase (November 2009 till May 2010) was V324 while the first processor supporting 669 the Operational Phase was V344. During 2011 version V500 was deployed and a full reprocessing of 670 the mission data set between January 2010 till October 2011 was carried out with a slightly modified 671 version V505. A new version V600 was ready by end of 2012, which, after further enhancements, 672 became V620 by early 2014. A second full mission reprocessing of the data between January 2010 and 673 May 2015 took place using V620, version which was deployed in early 2015. This section will focus 674 on the improvements brought in by V620, used in the second reprocessing, with respect to the earlier 675 V505 of the first reprocessing, as well as in the performance assessment of the new processor version. 


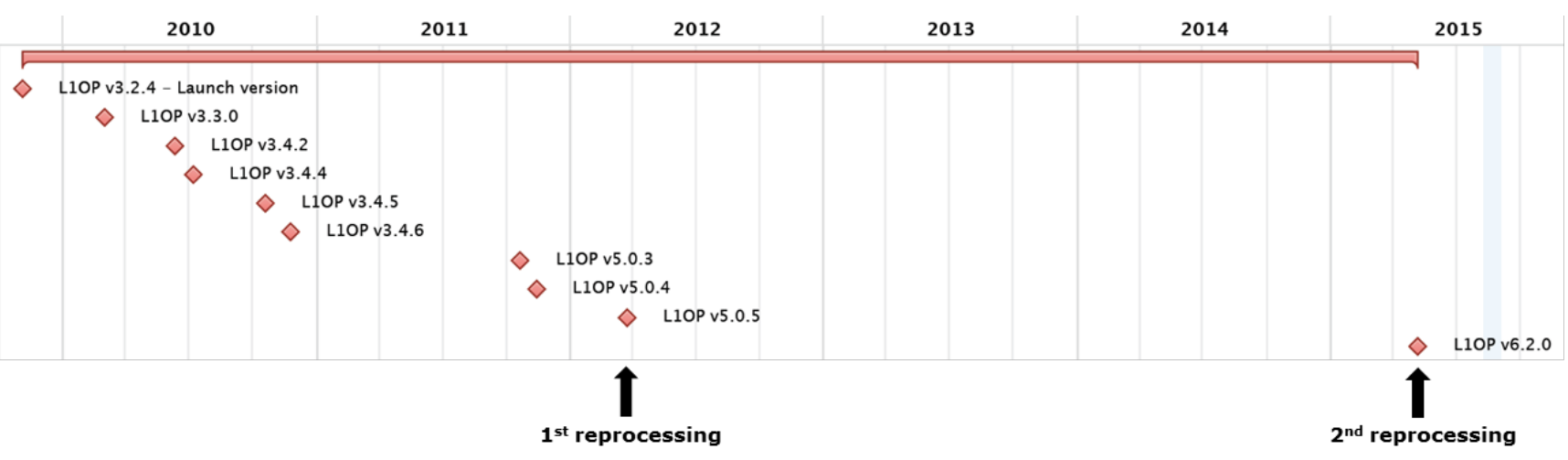

Figure 23: SMOS Level-1 data processor evolution

\subsection{Enhancements in Level-1 Processor Version V620 over V505}

\subsubsection{Fully Polarimetric Operation}

The equation below gives the matrix relation between the brightness temperature and the visibility vectors through the G observation matrix (Martín-Neira et al., 2002) (Corbella et al., 2004b):

$$
\left[\begin{array}{c}
\Delta V_{k j}^{H H} \\
\Delta V_{k j}^{V V} \\
\Delta V_{k j}^{H V}
\end{array}\right]=\left[\begin{array}{lll}
G_{T_{H H}}^{H H} & G_{T_{V V}}^{H H} & G_{T_{H V}}^{H H} \\
G_{T_{H H} V}^{V V} & G_{T_{V V}}^{V V} & G_{T_{H V} V}^{V V} \\
G_{T_{H H}}^{H V} & G_{T_{V V}}^{H V} & G_{T_{H V}}^{H V}
\end{array}\right] \times\left[\begin{array}{c}
\Delta T_{B}^{H H}(\xi, \eta) \\
\Delta T_{B}^{V V}(\xi, \eta) \\
\Delta T_{B}^{H V}(\xi, \eta)
\end{array}\right]
$$

The elements along the main diagonal of the $\mathrm{G}$ matrix involve co-polar antenna patterns. The elements outside the main diagonal include one or two cross-polar patterns. V505 was built using only co-polar patterns, that is, the G matrix was block-diagonal. The new version V620 makes use of the cross-polar patterns, which were measured on ground, and hence, implements a fully populated G matrix with all the blocks shown above. The first attempts to use the cross polar patterns were not successful though, because there was a sign inconsistency between the ground measurements and the Level-1 data processor. Once this issue was identified and solved, the expected performance improvement was achieved (Torres et al., 2015). The G matrix is used inside the Level-1 processor in the forward modelling as well as in the inverse process of image reconstruction (Khazaal et al., 2015). V620 is the first fully polarimetric SMOS Level-1 data processor. Among all enhancements, this one providing fully polarized data is perhaps the most significant one. In fact, this is the first time that fully polarized interferometric images from space are processed. 
REPLACE THIS LINE WITH YOUR PAPER IDENTIFICATION NUMBER (DOUBLE-CLICK

HERE TO EDIT) <

699 6.1.2 Use of the Relative Phase between Polarizations Measured on Ground

700

701

702

703

704

705

706

707

708

709

710

711

712

713

714

715

716

717

718

719

720

721

722

723

During the Image Validation Test (IVT) of MIRAS that was carried out on ground before launch, a set of 4 probes was placed in the ceiling of the Maxwell Electromagnetic Compatibility chamber of ESTEC to measure the relative phase between all LICEF receivers (Corbella et al., 2009). The instrument was operated in both dual and full polarization. For version V505 of the Level-1 processor, 2 separate sets of relative phases were retrieved from the IVT test: one with the relative phases for the horizontal polarization and another one for the vertical polarization. When preparing the next version, V620, of the processor, it became clear that the two sets of relative phases could have an offset between them which had to be corrected. Such phase bias across the two polarizations was in fact causing distortions in the Stokes-3 and, most clearly, Stokes-4 parameters. Therefore, the IVT data set was reanalyzed to determine the missing phase offset between the two polarizations, which was found to be of $-6.8^{\circ}$. This value was verified using Stokes-4 images over the ocean: as shown in Figure 24, the error (sigma displayed in the lower left corner) was indeed minimized for a phase offset close to the retrieved one. All phases corresponding to vertical polarization were then reduced by that amount in version V620 of the processor.

$$
\Theta_{x y}=0^{\circ}
$$

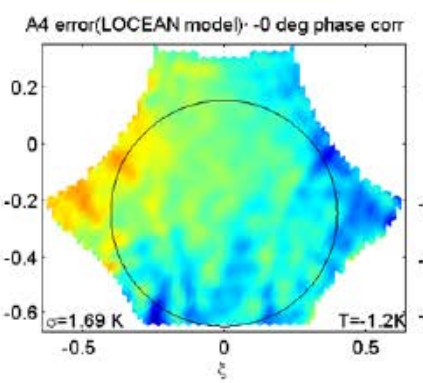

$\Theta_{x y}=-5^{\circ}$

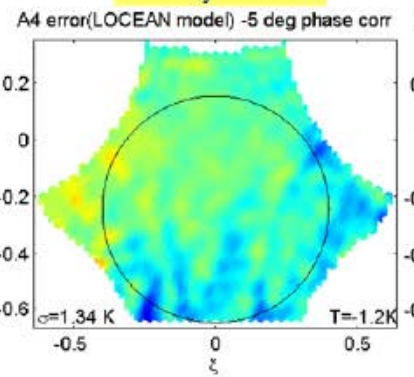

$$
\Theta_{x y}=-10^{\circ}
$$

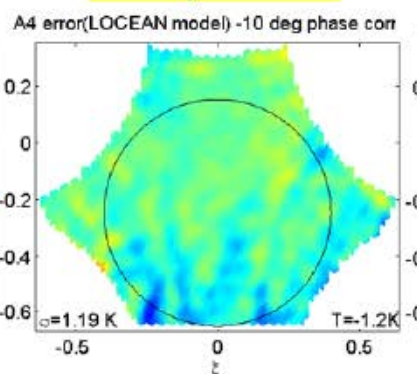

$\Theta_{x y}=-15^{\circ}$

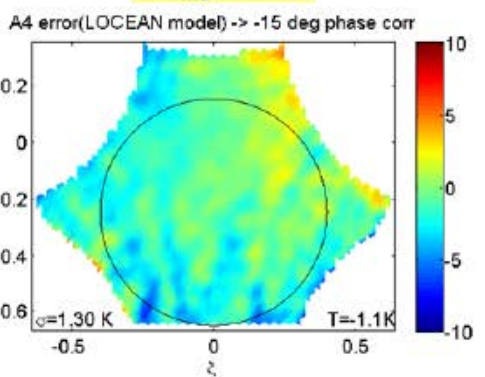

Figure 24: Validation of the relative phase between polarizations using Stokes-4 over ocean.

\subsubsection{Use of Average Antenna Patterns across 3 Frequencies Measured on Ground}

The antenna patterns of all and every element of MIRAS embedded in the array were carefully measured in an antenna test range. The measurements were performed at the center frequency, 1413.5 $\mathrm{MHz}$, as well as at the band edges, 1404 and $1423 \mathrm{MHz}$. In version V505 of the Level-1 processor only 
REPLACE THIS LINE WITH YOUR PAPER IDENTIFICATION NUMBER (DOUBLE-CLICK HERE TO EDIT) $<$

724 the patterns at the center frequency were used. In version V620, the average antenna pattern across the 7253 measured frequencies is used, which reduces slightly the spatial ripple, as shown in the left lower corner of each panel of Figure 25.
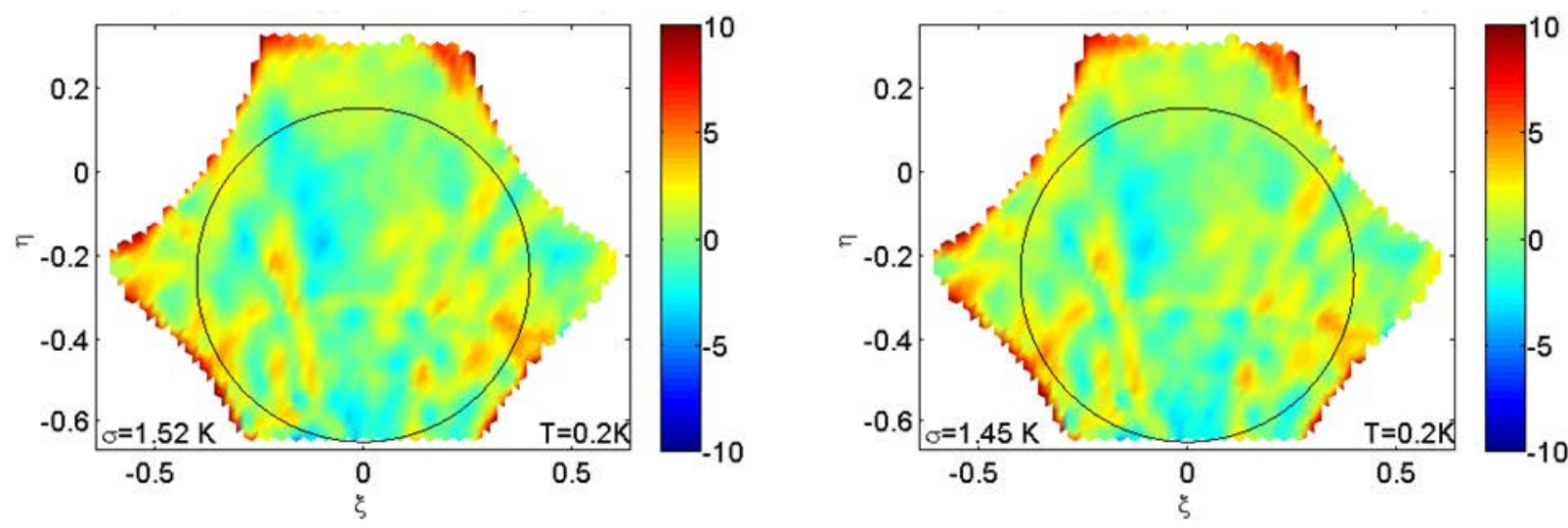

727

728

729

730

731

732

733

734

735

736

737

738

739

740

741

742

743 744 the left panel of Figure 25.

Figure 25: Deviation images from an ocean model in X-polarization using 1 pattern (left) or the average of the 3 measured patterns on ground (right) -similar results were obtained in Y-polarization-.

\subsubsection{Replacement of Antenna Pattern of Hinge Elements by their Neighbour's}

Analyzing in detail the measured antenna patterns, it was noticed that those corresponding to elements next to one of the hinges of the deployed arms (upper left panel of Figure 26), presented some ripples which the elements in the center of the arm segments did not exhibit (upper center panel). Some research led to the conclusion that these ripples were caused by travelling waves between the arm and the supporting structure that was used to hold it during the measurements, as shown in the lower left panel of Figure 26, which would leak out through the next hinge and segment contour causing a typical interference pattern. Since the instrument in flight configuration does not have any supporting structure in the back, the real patterns of the hinge elements should be free of fringes and similar to those of the central elements in each arm segment. For this reason, in the Level-1 processor version V620, the antenna patterns of the hinge elements was replaced by that of its inner neighbours, which reduces slightly the spatial ripple of the images, as illustrated in the right panel of Figure 26, to be compared to 


\section{REPLACE THIS LINE WITH YOUR PAPER IDENTIFICATION NUMBER (DOUBLE-CLICK HERE TO EDIT) <}

747 From Figure 22, it is seen that the NIR-CA unit (bottom panels) is the most stable of the 3 Noise 748 Injection Radiometers of MIRAS. To assure the best temporal stability, it was decided to use, in V620, 749 only the antenna temperature measured by NIR-CA, and not those from NIR-AB and NIR-BC, for the 750 visibility sample at the origin $\mathrm{V}(0,0)$. All 3 NIR units are still employed in the measurements of other 751 visibility samples outside the origin as well as in the measurement of the noise diodes of the on-board 752 Calibration System.

753

754

755

756

757

758

759

760

761

762

763

764

765
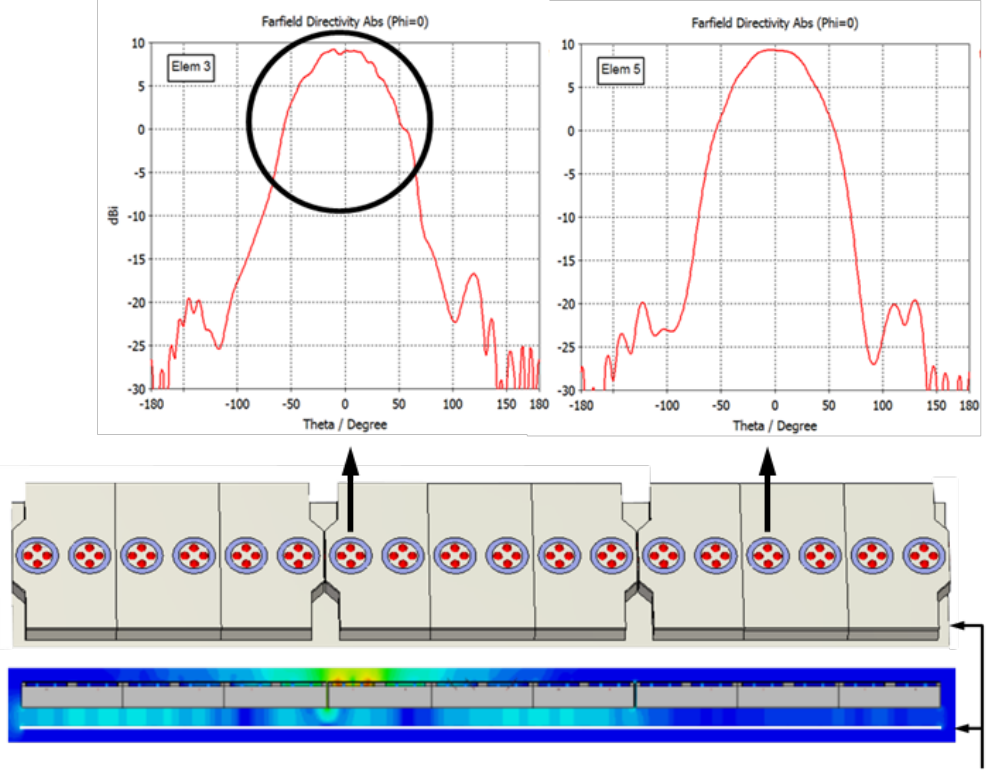

Supporting structure

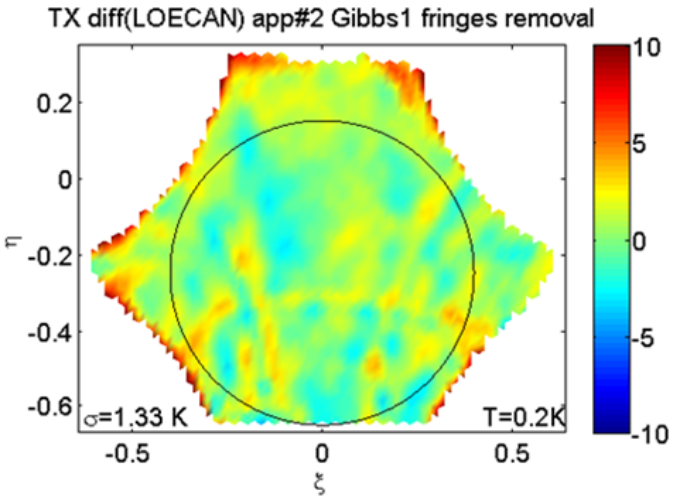

Figure 26: Antenna patterns of elements next to a hinge (top left) and away from it (top center); electromagnetic simulation showing leakage between arm and back supporting structure (left bottom); image obtained by replacing hinge patterns by their neighbour's (right).

\subsubsection{Use of In-Orbit Antenna Loss}

As mentioned earlier, antenna losses L1+L2 could not be accurately measured on ground due to set up uncertainties, and instead, were characterized in orbit, for V620 of the Level-1 processor, thanks to the more favourable external calibration manoeuvres (refer to Figure 16). The final estimation of the antenna loss was carried out after removing those instances with detector voltage fluctuations as the examples in Figure 13. Once the total loss had been measured, a second step, critical for the temporal stability, was performed, that of determining the L1 and L2 separately for the Noise Injection Radiometers. As the external calibration could only provide the ensemble loss, the split into its two 
REPLACE THIS LINE WITH YOUR PAPER IDENTIFICATION NUMBER (DOUBLE-CLICK HERE TO EDIT) $<$

768 contributions was done by minimizing the orbital variations, taking as metrics the descending minus

769 ascending deviations over the ocean with respect to a forward model. Such exercise resulted in a 770 minimum of orbital variation for $\mathrm{L} 1=0.15 \mathrm{~dB}$ as illustrated in Figure 27, the remaining of the total loss

771 measured in orbit being assigned to L2. The values used in V620 for the L2 antenna loss of each 772 receiver and polarization are constant (do not change over time) and equal to their average value 773 obtained across all external calibrations, using the optimized $\mathrm{L} 1=0.15 \mathrm{~dB}$. This procedure to split the 774 antenna losses in its two contributors L1 and L2 was based on the much stronger relationship of 775 L1 with orbital variations than L2, L1 being tightly influenced by the skin temperature of the 776 antenna.

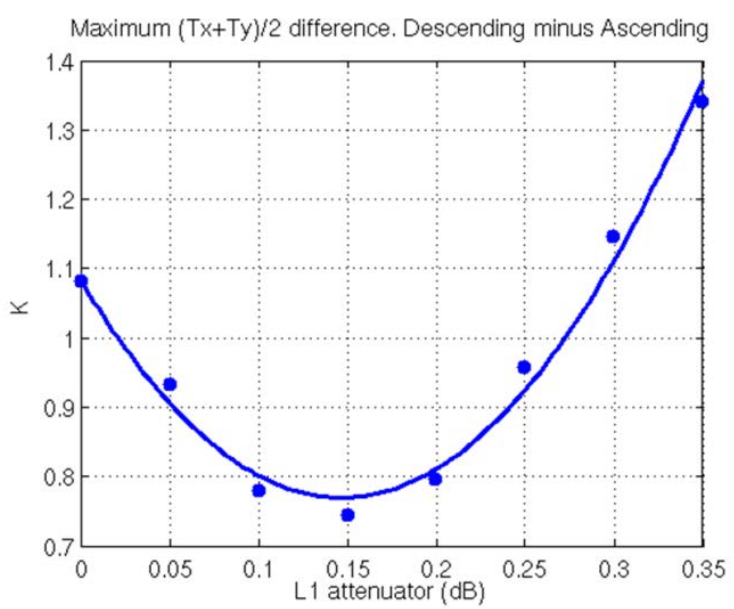

Figure 27: Split of NIR antenna losses achieved by minimizing the descending minus ascending pass difference of the deviations of the first Stokes parameter (divided by 2) from a model of the brightness temperature of the ocean (in Kelvin)

\subsubsection{Improved Gibbs-1 Image Reconstruction}

In addition to being fully polarimetric, as commented earlier, the image reconstruction of V620, depicted in Figure 28, brings two other main improvements over V505. The first one is the implementation of the whole processing on hexagonal grids, as opposed to rectangular ones, to avoid interpolation errors. The second consists of a new Gibbs-1 'model approach' by which the image reconstruction is performed over residual visibilities resulting from subtracting the estimated contribution of the Corbella term, the sky and the Earth, from the measured visibilities. 


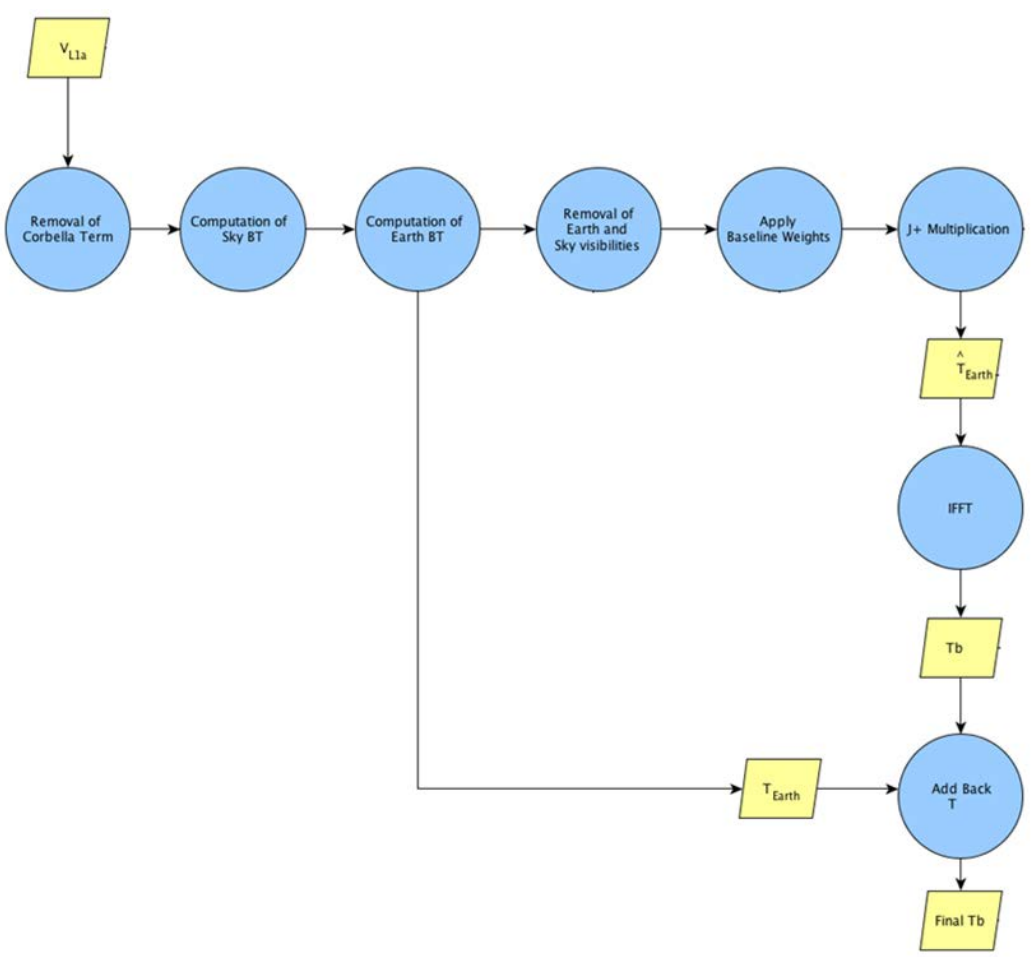

\subsubsection{Improved Removal and Flagging of Sun and RFI sources}

Figure 28: Improved Gibbs-1 model approach for image reconstruction as implemented in V620.

798 Sun brightness temperature is estimated through a 4-point spatial interpolation instead of taking the 799 single closest neighbour point, this allowing a much finer positioning of the energy and thus, a 800 reduction of the Sun tails. Several enhancements were also done to the flagging of the pixels affected 801 by the Sun brightness temperature, in particular, the dynamic adjustment of the width of the tails of the 802 real Sun and its aliases for every snapshot, and the correct flagging of all tails (one of the tails was not 803 properly flagged in the previous version), as shown in Figure 29. As for the flagging and treatment of 804 RFI sources and their impact, the improvements are reported in (Oliva et al., in press) (Khazaal et al., 805 2014) (Daganzo-Eusebio et al., 2013). 
819 the antenna and the brightness temperatures, systematic spatial errors in the images (bias and ripple),

820 Sun correction, land-sea contamination and random noise. The Cold Sky, the Pacific Ocean and

821 Antarctica are the targets where the metrics have been evaluated, by comparing observations to a 822 surveyed map of the sky, a radiative transfer model of the ocean (Ocean Target Transformation 823 or OTT) or simply their average value over Antarctica (Ice Target Transformation or ITT) 824 respectively.

Figure 29: SMOS extended alias-free field of view showing, in grey, the pixels which are flagged due to the Sun tails (real Sun is on the right, with 2 alias on left top and left bottom). The width of the tails with flagged pixels has been increased with the improved Sun tails dynamic flagging of V620, and all tails are now flagged (note the lower right corner tail in V6).

\subsection{Performance of Level-1 Processor Version V620}

This section is devoted to present the performance of the currently operational version of the Level-1 processor, V620, in comparison with the earlier version V505. The performance has been assessed over the entire data set of the two reprocessing campaigns, one with each processor version. The quantification of the performance has been carried out following some defined metrics, as depicted in Figure 30, comprising: calibration parameters, temporal stability (orbital, seasonal and yearly) of both
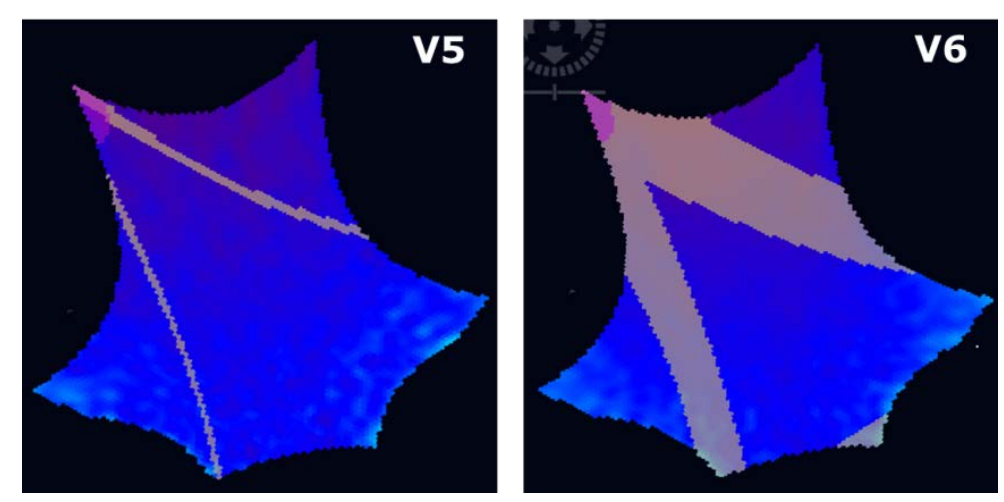
rectively. 
HERE TO EDIT) <

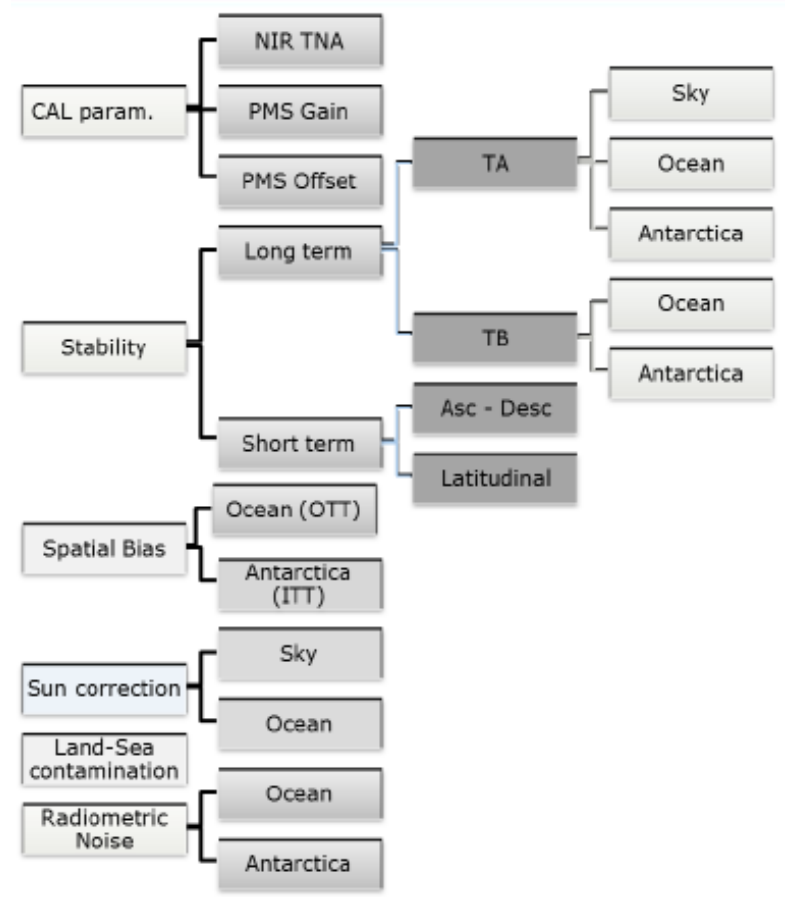

Figure 30: Overview of the metrics used to evaluated the performance of the Level-1 processor.

\subsubsection{Removed Negative Slope at High Elevation Angles}

V505 suffered from a negative slope in the dependence of the brightness temperature with elevation angle, in a way that measurements at low incidence were too cold when compared against modeled values. This is illustrated in the left panel of Figure 31 which shows the brightness temperature measured by SMOS, in horizontal and vertical polarizations, as a function of incidence angle over Dome-C station in Antarctica (Macelloni et al., 2013). As reference, the red dashed line represents a model, the diamonds are Aquarius observations and the solid circles ground measurements. Besides the discrepancy at high incidence angles which is expected due to the unavoidable sky contamination in the ground observations (through the part of the main lobe above the ice horizon), the SMOS brightness temperatures are clearly colder than the model towards $0^{\circ}$ incidence. The enhancements implemented in V620 described earlier (in particular in Section 6.1.7) resulted in a substantial reduction of such cold trend at low incidence, as can be seen in the right panel of Figure 31 . It is also worth noting that, for V620, the ripples along incidence angle are smaller and the match with Aquarius and Dome-C ground radiometer is better. 
REPLACE THIS LINE WITH YOUR PAPER IDENTIFICATION NUMBER (DOUBLE-CLICK HERE TO EDIT) <
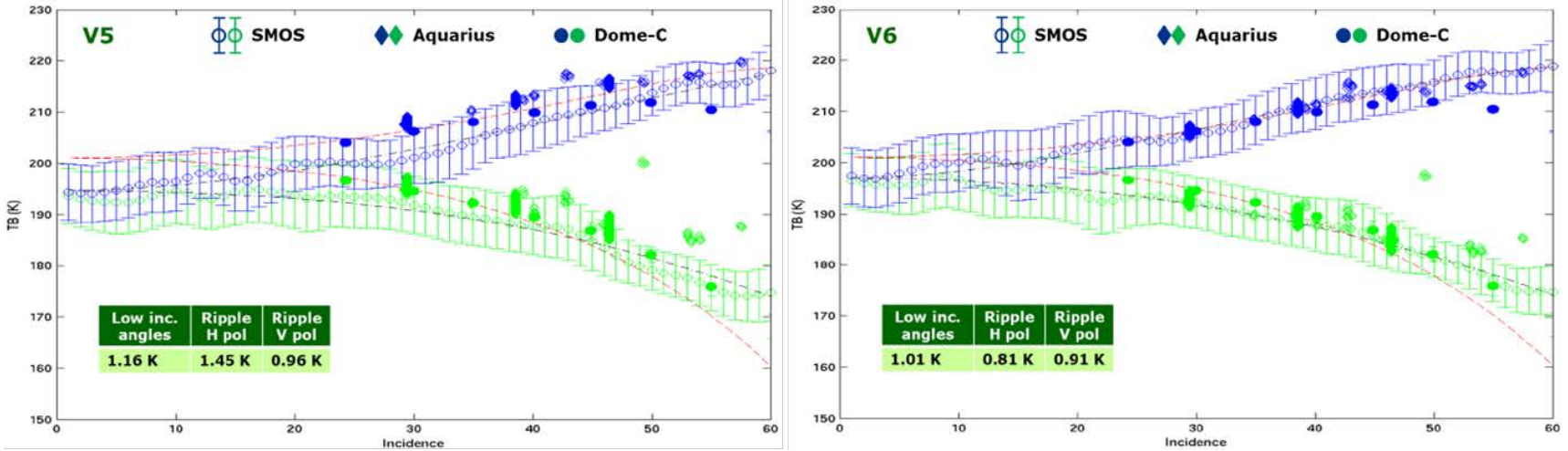

Figure 31: Performance of V505 (left) and V620 (right) with incidence angle over Antarctica.

\subsubsection{Lower Spatial Ripple}

Although there is a limit to how much the spatial ripple can be removed as explained before, Figure 32 shows that V620 achieves about $0.2 \mathrm{~K}$ lower spatial ripple in both polarizations than V505, thanks to

the improvements in Sections 6.1.1 through 6.1.4 and 6.1.7. The root mean square spatial ripple of V620 over most of the Extended Alias-Free Field of View is therefore of about 1.5 and $2.0 \mathrm{~K}$ for X and Y polarizations respectively, evaluated over the ocean. It has to be noted that the bias of V620 is warmer than that of V505, overshooting almost $1 \mathrm{~K}$ in X polarization above the forward ocean model.
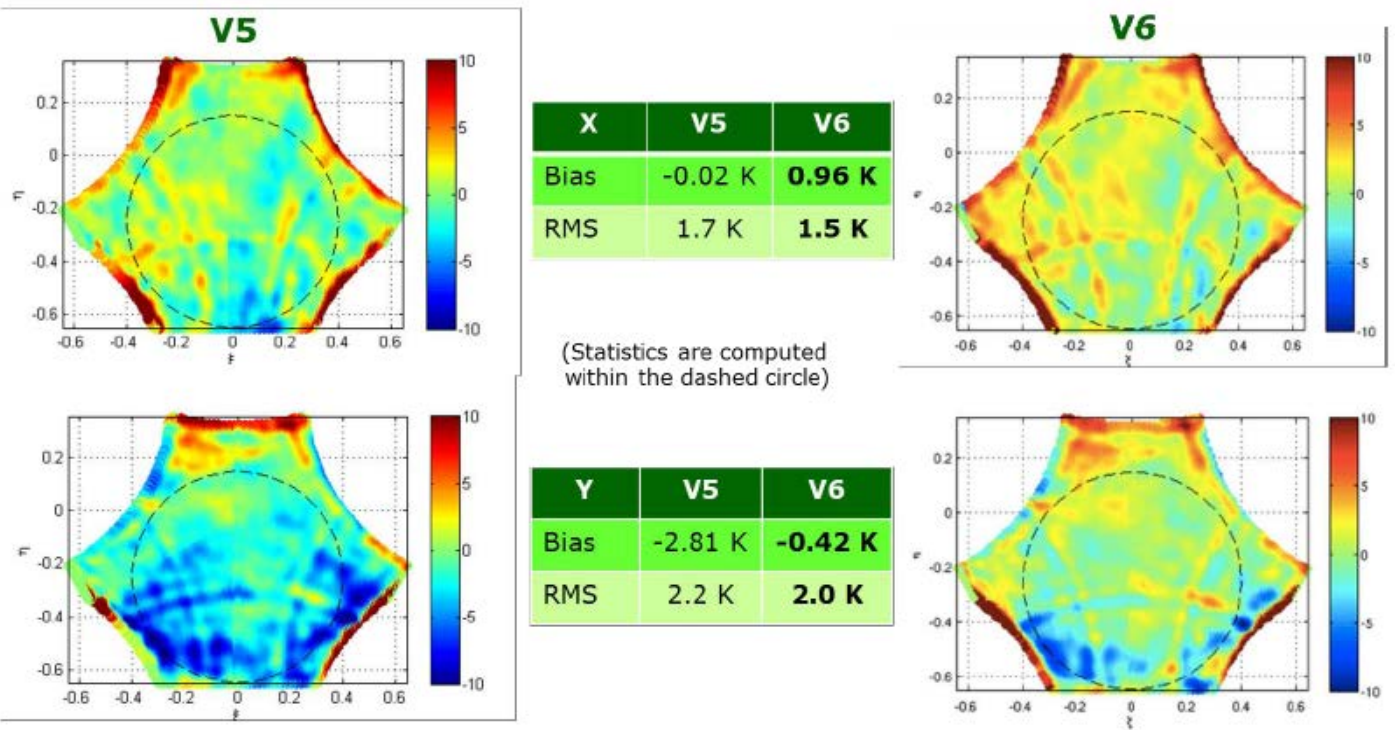

Figure 32: Spatial ripple and bias of V505 (left) and V620 (right) over Ocean (colour scale in Kelvin)

\subsubsection{Improved Stokes-3 and Stokes-4 Parameters}


REPLACE THIS LINE WITH YOUR PAPER IDENTIFICATION NUMBER (DOUBLE-CLICK HERE TO EDIT) <

860 The fully polarimetry operation of V620 including the cross-polar patterns with corrected sign 861 convention (Section 6.1.1) and relative phase between polarizations (Section 6.1.2) yield significant improvements in the Stokes-3 and Stokes-4 parameters (Lin et al., 2013). This is clearly shown in Figure 33, where more uniform residuals against the ocean model are obtained with V620 (right column) than with V505 (left column). The cleaner polarimetric brightness temperatures provided by V620 allows accurate estimation of the ionospheric total electron content and the Faraday rotation angle directly from SMOS observations (Corbella et al., 2015).

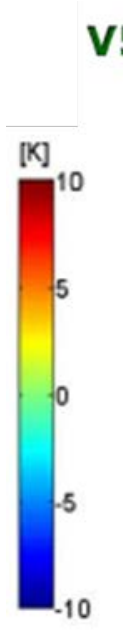

Figure 33: Residual Stokes-3 (top) and Stokes-r4 (bottom) of V
(colour scale in Kelvin)
V6

$\mathrm{T} 3 \equiv \operatorname{Re}\{\mathrm{T} x \mathrm{y}\}$

$\mathrm{T} 4 \equiv \operatorname{Im}\{\mathrm{T} x y\}$

\subsubsection{Removed Latitudinal Drift}

The red line in Figure 34 shows the deviation of the Stokes-1/2 parameter (average of the vertical and horizontal brightness temperatures) against a forward model along a strip of the Pacific Ocean from $50^{\circ}$ South to $20^{\circ}$ North, for version V620. The plot is for one orbit, but the general behaviour is systematic, with some seasonal variations as shown in the Hövmoller plot in the right panel of

Figures 6. Besides some variations on each side of the equator which can be related to geophysical signals (unmodeled rain and surface roughness effects), there is no tendency with latitude as expected, thanks mainly to the improvement in Section 6.1.6. This is an important improvement 
REPLACE THIS LINE WITH YOUR PAPER IDENTIFICATION NUMBER (DOUBLE-CLICK HERE TO EDIT) <

over V505 processor, for which the Stokes-1/2 parameter, shown by a blue line on the same figure, did have a significant slope over latitude.

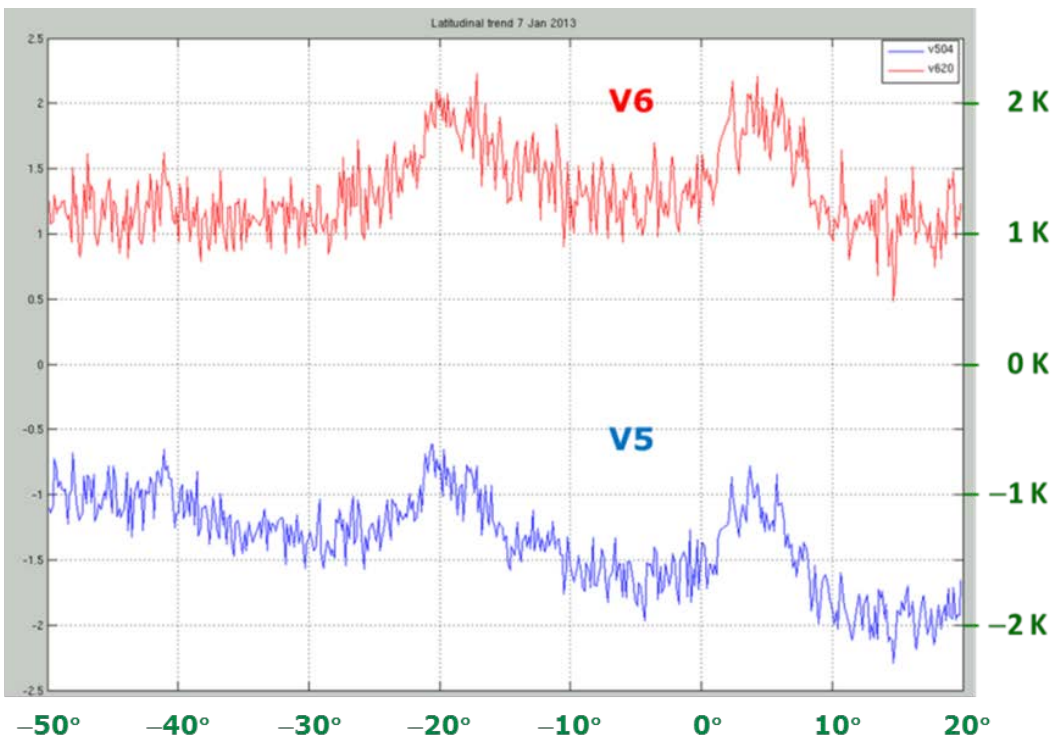

Latitude

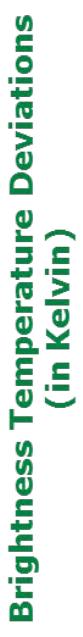

Figure 34: Latitudinal trend of the brightness temperature deviation from an ocean model, averaged in the alias-free field of view, of V505 (blue line), and corrected value in V620 (red line).

\subsubsection{Reduced Orbital Variations}

The reduced orbital variations of V620 in the Stokes-1/2 parameter over the ocean have already been introduced and shown in the right panel of Figure 7 . What is presented in the left panel of the same figure is the strong orbital variations, of about $2 \mathrm{~K}$ peak to peak, that V505 exhibited between ascending and descending passes in the same Stokes-1/2 parameter. These pronounced variations have been reduced in V620 mostly thanks to the optimization and use of the antenna losses measured in orbit (Section 6.1.6) and the use of only the most stable NIR unit (Section 6.1.5).

\subsubsection{Improved Yearly and Seasonal Stability}

Figure 6 shows the latitudinal, seasonal and yearly variations of the Stokes-1/2 parameter over the ocean. The left plot, for V505, has strong variations with latitude which, as discussed above, have been removed in V620, shown in the right panel. V505 presents also significant seasonal variations, seen as $\pm 2 \mathrm{~K}$ alternating blue and red bands in the corresponding Hovmöller plot. These strong seasonal 
REPLACE THIS LINE WITH YOUR PAPER IDENTIFICATION NUMBER (DOUBLE-CLICK HERE TO EDIT) <

903 fluctuations have been greatly reduced in V620, down to $\pm 0.4 \mathrm{~K}$, thanks to the improvement in 904 Section 6.1.6. In addition, a $-0.18 \mathrm{~K} /$ year cooling yearly drift is present in V505 data, which has 905 disappeared in V620, for which the trend is of only $-0.003 \mathrm{~K} /$ year. The long term stability of V620 has 906 further been verified over Antarctica, as presented in Figure 35, which shows the vertical and 907 horizontal brightness temperature at $42^{\circ}$ incidence angle measured by SMOS for a period of over 5 908 years. The fluctuations in horizontal polarization are known to be due to surface roughness fluctuations, 909 being the vertical polarization the best indicator to check for the instrument long term stability. As it 910 can be seen, the yearly drift is indeed negligible.

911

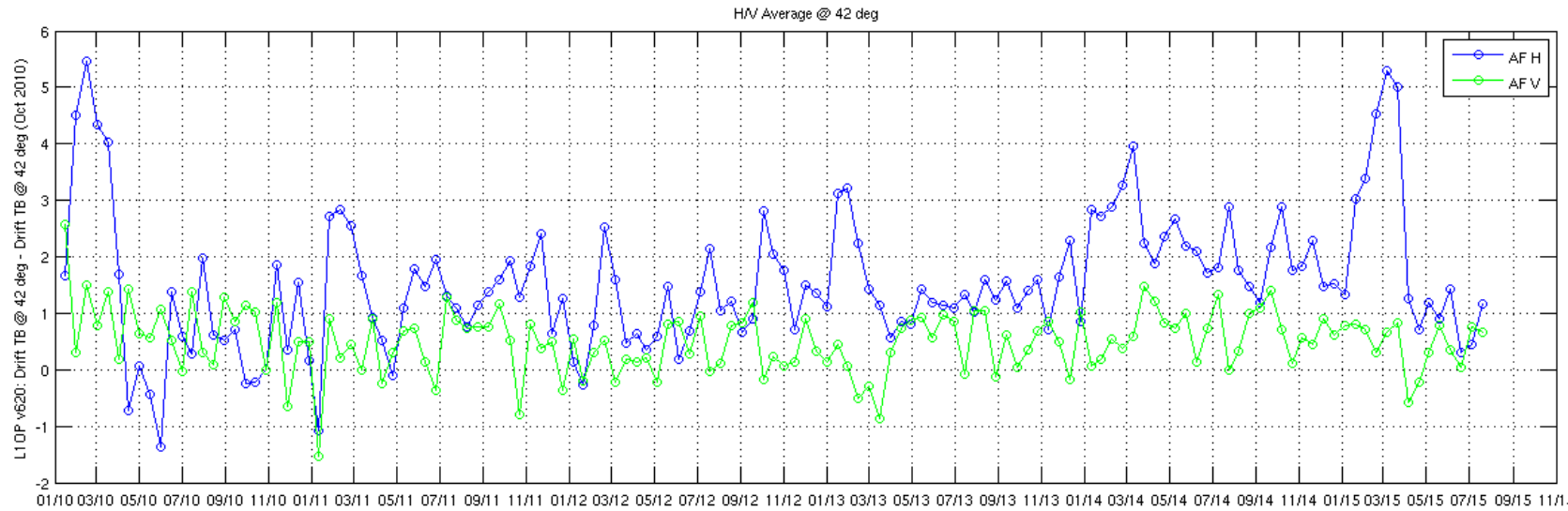

Figure 35: Over 5-year record of vertical and horizontal SMOS brightness temperatures at $42^{\circ}$ incidence angle within the Alias-Free Field of View over Antarctica.

\section{CURRENT INVESTIGATIONS}

Despite the major improvements brought in by V620 version of the SMOS Level-1 processor over the previous version V505 which have been presented, several remaining challenges require further work, in particular, the residual orbital and seasonal variations, including the eclipse season, the further reduction of the spatial ripple and the Sun tails, not to forget the detection and mitigation of RFI effects. This section presents some of the on-going investigations to advance in these directions.

\subsection{Level-1 Processor Version V700}


REPLACE THIS LINE WITH YOUR PAPER IDENTIFICATION NUMBER (DOUBLE-CLICK HERE TO EDIT) <

925 As mentioned in the introduction a new version of the processor, V700, has already been delivered, 926 whose major change with respect to the currently operational version V620 is that it can run a so-called 927 'All-LICEF' mode. The implementation of the All-LICEF mode has, as objective, the simplification of 928 the overall calibration procedure of the MIRAS instrument, to achieve better calibrated measurements 929 and to make faster progress in correcting some of the remaining spatial errors and temporal variations. 930 The concept behind the All-LICEF mode is depicted in Figure 36. The upper part shows the man 931 calibration steps followed in V620: first the NIR radiometers are calibrated using the Cold Sky and an 932 internal matched load, to provide the visibility sample at the origin $\mathrm{V}(0,0)$; then NIR is used to calibrate 933 the noise diodes of the on-board Calibration System (CAS); finally the CAS system calibrates all the 934 LICEF receivers of MIRAS. The lower part of Figure 36 shows instead, the much simpler and direct 935 calibration approach of the All-LICEF mode: the LICEF receivers are directly calibrated using the Cold 936 Sky and an internal matched load, and hence, the visibility sample at the origin can be measured by 937 averaging all the calibrated antenna temperatures of the LICEF receivers. The straight advantage of the 938 All-LICEF mode is therefore its extreme simplicity. This mode of operation was already envisaged 939 well before launch, but only the accumulation of 5 years of flight experience allows to properly 940 assessed its performance. It has to be noted that while the Noise Injection Radiometers are intrinsically 941 more stable than the LICEF total power radiometers, the fact that there are 69 of the latter and only 3 of 942 the former (in fact only the most stable NIR unit is currently used), offsets the final result in favour of 943 the All-LICEF solution. To show one example, Figure 37 shows the temporal variations for V620 (left 944 panel) and V700 in All-LICEF mode (right panel), and it can be noticed that the warming trend of 2014 945 for V620 is not so pronounced in V700. Another illustration of the benefits of the All-LICEF mode is 946 given in Figure 5, where the land-sea contamination correction based on the adjustment of the 947 correlator efficiency coefficients explained earlier has been successfully tested with V700 (right panel) 948 by comparison with the current V620 (left panel) where a significant coastal signature is still 
REPLACE THIS LINE WITH YOUR PAPER IDENTIFICATION NUMBER (DOUBLE-CLICK HERE TO EDIT) <

949 observable. The All-LICEF branch of V700 is undergoing through a detailed assessment before it is 950 accepted for operational use.
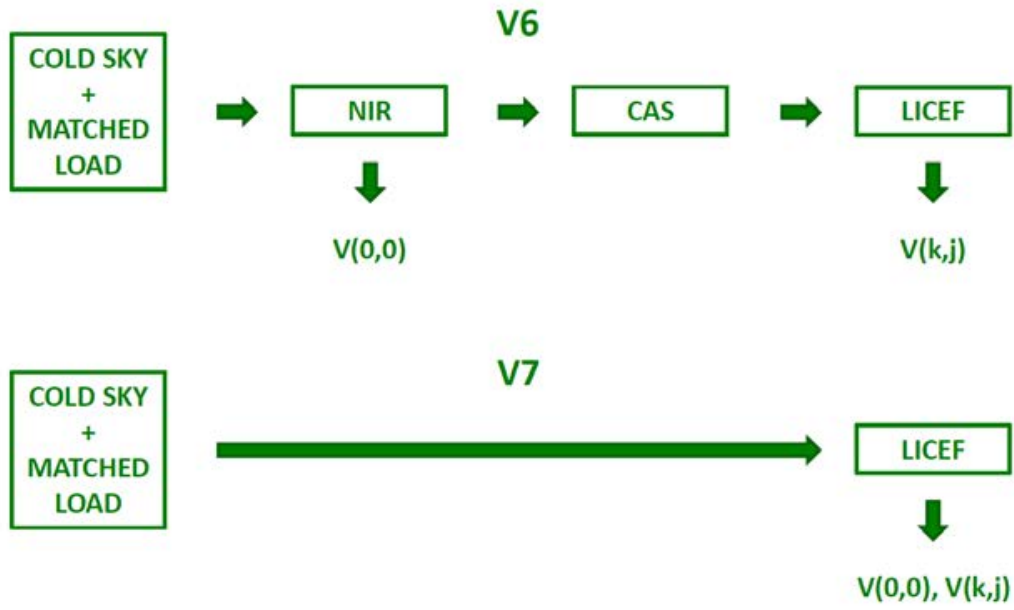

Figure 36: All-LICEF mode

\section{$955 \quad 7.2 \quad$ Beyond V700}

956 Other improvements beyond version V700 are in the pipeline, namely the implementation of an 957 improved Gibbs-2 based image reconstruction algorithm, the further elaboration of improved spatial 958 ripple and Sun mitigation techniques, as well the reduction of residual temporal fluctuations.

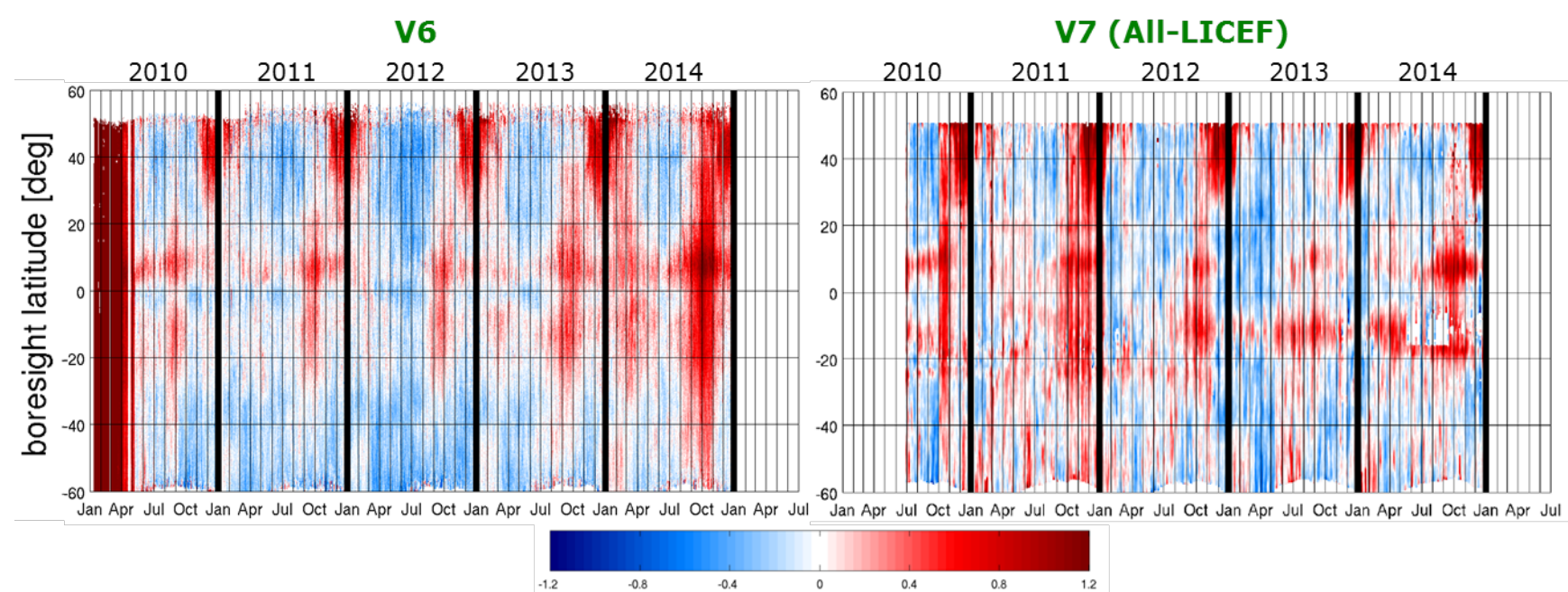

961 Figure 37: Descending-minus-Ascending pass Stokes-1/2 parameter over the Pacific Ocean, averaged 962 in the alias-free area, with V620 (left) and V700 in All-LICEF mode (right) -colour scale in Kelvin- 
REPLACE THIS LINE WITH YOUR PAPER IDENTIFICATION NUMBER (DOUBLE-CLICK HERE TO EDIT) <

\section{CONCLUSIONS}

SMOS mission has passed its extended life time of 5 years and continues to deliver good quality data, with both the MIRAS payload and the PROTEUS platform being in good health. The accumulated data

969 record has allowed continued insight and improvement, from launch till today, of the MIRAS 970 instrument and the Level-1 processor which transforms the visibility measurements into brightness 971 temperature images. This paper has described the results of the detailed investigation of the calibration 972 data and images, in various aspects, including instrumental behaviour and image reconstruction. 973 Special focus has been given to the presentation of the performance of the currently operational version 974 of the Level-1 processor, V620, and its improvements by comparison to the earlier version V505. V620 975 is a fully polarimetric processor accounting for cross polar terms, with an enhanced image 976 reconstruction technique and based on an improved in-orbit calibration approach which has resulted in 977 overall cleaner and more stable brightness temperature images. Some hints have also been given about 978 on-going investigations with a new processor version already delivered, V700, featuring the All-LICEF 979 mode with a much simpler calibration strategy with potential to reduce the land-sea contamination.

980 Finally, some future avenues, as the Gibbs- 2 method, have been mentioned, addressing the still 981 remaining challenges as the residual orbital and seasonal fluctuations, spatial ripple and Sun correction.

\section{ACKNOWLEDGEMENTS}

The authors would like to express their thanks to Mariano Kornberg with ESA, for providing the long series of instrument parameter values shown in the paper.

\section{REFERENCES}

Anterrieu E., P. Waldteufel, A. Lannes, (2002), “Apodization Functions for 2-D Hexagonally Sampled Synthetic Aperture Imaging Radiometers", IEEE Transactions On Geoscience and Remote Sensing, Vol. 40, No. 12, pp.47-51.

Anterrieu E., (2007), "On the Reduction of the Reconstruction Bias in Synthetic Aperture Imaging Radiometry", IEEE Transactions On Geoscience and Remote Sensing, Vol. 45, No. 3, pp. 592-601. 


\section{REPLACE THIS LINE WITH YOUR PAPER IDENTIFICATION NUMBER (DOUBLE-CLICK HERE TO EDIT) <}

Anterrieu E., M. Suess, F. Cabot, P. Spurgeon, A. Khazaal, (2015), "An additive mask correction approach for reducing the systematic floor error in imaging radiometry by aperture synthesis", IEEE Geoscience and Remote Sensing Letters, 12(7), pp. 1441-1445.

Brown M.A., F. Torres, I. Corbella, A. Colliander, (2008), "SMOS Calibration", IEEE Transactions On Geoscience And Remote Sensing, Vol. 46, No. 3, 646 - 658.

Camps A., (1996), “Application of Interferometric Radiometry to Earth Observation”, PhD Thesis, Polytechnic University of Catalonia.

Camps A., M. Vall-llossera, N. Duffo, M. Zapata, I. Corbella, F. Torres, (2004), "Sun Effects in 2-D Aperture Synthesis Radiometry Imaging and Their Cancelation", IEEE Transactions On Geoscience and Remote Sensing, Vol. 42, No. 6, pp.1161-1167.

Camps A., I. Corbella, F. Torres, N. Duffo, M. Vall-llosera, M. Martín-Neira, (2005), “The Impact of Antenna Pattern Frequency Dependence in Aperture Synthesis Microwave Radiometers", IEEE Transactions On Geoscience and Remote Sensing, Vol. 43, No. 10, pp. 2218-2224.

Camps A., M. Vall-llossera, I. Corbella, N. Duffo, F. Torres, (2008), "Improved Image Reconstruction Algorithms for Aperture Synthesis Radiometers", IEEE Transactions On Geoscience and Remote Sensing, Vol. 46, No. 1, pp.146-158.

Chiuderi Drago F., M. Felli and G. Tofani, 1977, High resolution intensity and polarization structure of the Sun at $21 \mathrm{~cm}$, Astron. Astrophysics. 61, 79-91.

Colliander A., L. Ruokokoski, J. Suomela, K. Veijola, J. Kettunen, V. Kangas, A. Aalto, M. Levander, H. Greus, M. Hallikainen, J. Lahtinen, (2007), "Development and Calibration of SMOS Reference Radiometer", IEEE Transactions On Geoscience and Remote Sensing, Vol. 45, No. 7, pp. 1967-1977.

Colliander, E. Dinnat, D. Le Vine, C.-S. Chae, and J. Kainulainen, 2015, "Assessing long-term stability of SMOS zero-baseline antenna temperature using the Aquarius antenna temperature simulator," Geoscience and Remote Sensing Letters, IEEE, vol. 12, no. 8, pp. 1680-1684.

Corbella I., N. Duffo, M. Vall-llossera, A. Camps, and F. Torres, (2004a), "The visibility function in interferometric aperture synthesis radiometry", IEEE Transactions On Geoscience and Remote Sensing, Vol. 42, No. 8, pp. $1677-1682$.

Corbella I., (2004b), "Polarimetric and Extended Formulation of the Visibility Equation". MIRAS Demonstrator Pilot Project 3, MP3-TN-UPC-3000-0002 v1.1, ESA Contract 15138/01/NL/SF.

Corbella I., F. Torres, N. Duffo, M. Martín-Neira, V. González-Gambau, A. Camps, M. Vall-1lossera, (2009), "On-Ground Characterization of the SMOS Payload", IEEE Transactions on Geoscience and Remote Sensing, Volume 47, Issue 9, pages: 3123 - 3133.

Corbella I., F. Torres, N. Duffo, I. Durán, M. Pablos, M. Martín-Neira, (2012), "Enhanced SMOS Amplitude Calibration Using External Target", Proceedings of IGARSS-2012, Munich (Germany).

Corbella I., F. Torres, L. Wu, N. Duffo, I. Durán, M. Martín-Neira, (2014), "SMOS Image Reconstruction Errors", poster presented at IGARSS-2014, Quebec (Canada).

Corbella I., L. Wu, F. Torres, N. Duffo, M. Martín-Neira, (2015), "Faraday Rotation Retrieval Using SMOS Radiometric Data”, IEEE Transactions On Geoscience and Remote Sensing, Vol. 12, No. 3, pp. 458-461. 


\section{REPLACE THIS LINE WITH YOUR PAPER IDENTIFICATION NUMBER (DOUBLE-CLICK HERE TO EDIT) <}

1048

1049

1050

1051

1052

1053

1054

1055

1056

1057

1058

1059

1060

1061

1062

1063

1064

1065

1066

1067

1068

1069

1070

1071

1072

1073

1074

1075

1076

1077

1078

1079

1080

1081

1082

1083

1084

1085

1086

1087

1088

1089

1090

1091

1092

1093

1094

1095

1096

1097

1098

1099

1100

1101

1102

Corbella I., I. Duran, L. Wu, F. Torres, N. Duffo, M. Martín-Neira, (in press), "Impact of correlator efficiency errors on SMOS land-sea contamination", submitted to the Transactions on Geoscience and Remote Sensing in January 2015.

Daganzo-Eusebio, E.; Oliva, R.; Kerr, Y. H.; Nieto, S.; Richaume, P.; Mecklenburg, S., (2013), "SMOS Radiometer in the 1400-1427-MHz Passive Band: Impact of the RFI Environment and Approach to Its Mitigation and Cancellation," Geoscience and Remote Sensing, IEEE Transactions on, vol. 51, no. 10, pp. 49995007 .

Díez-García R., M. Martín-Neira, (2014), "Analysis and Characterization of the Reconstruction Error in Interferometric Synthetic Aperture Imaging Radiometers”, MicroRad-2014, Pasadena (California, US).

González-Gambau, V., Turiel, A., Olmedo, E., Martínez, J., Corbella., I., and Camps, A., (2015), 'Nodal sampling: a new image reconstruction algorithm for SMOS', IEEE Transactions on Geoscience and Remote Sensing, in press, doi: 10.1109/TGRS.2015.2499324.

Gourrion J., S. Guimbard, M. Portabella, R. Sabia, (2013), "Toward an Optimal Estimation of the SMOS Antenna-Frame Systematic Errors", IEEE Transactions on Geoscience and Remote Sensing, Vol. 51, No. 9, pp.4752-4760.

Kainulainen J., A. Colliander, (2010), "In-Orbit Performance of the SMOS Reference Radiometers - Results from the Early Commissioning Phase", MicroRad-2010, Washington (US).

Kainulainen J., A. Colliander, J. Closa, M. Martín-Neira, R. Oliva, G. Buenadicha, P. Rubiales-Alcaine, A. Hakkarainen, M. Hallikainen, (2012), "Radiometric Performance of the SMOS Reference RadiometersAssessment After One Year of Operation", IEEE Transactions on Geoscience and Remote Sensing, vol. 50, no. 5, pp. 1367-1383.

Khazaal A., H. Carfantan, E. Anterrieu, (2009), "On the Reduction of the Systematic Error in Imaging Radiometry by Aperture Synthesis: a New Approach for the SMOS Space Mission", IEEE Transactions on Geoscience and Remote Sensing, Vol. 6, No. 1, pp.47-51.

Khazaal, A., Cabot, F., Anterrieu, E., Soldo, Y. (2014), "A Kurtosis-Based Approach to Detect RFI in SMOS Image Reconstruction Data Processor". IEEE Transactions on Geoscience and Remote Sensing, Volume 52, Issue 11, pp.7038-7047.

Khazaal, A., Leroux, D.J., Cabot, F., Richaume, P., Anterrieu, E., (2015), "Effect of the Polarization Leakage on the SMOS Image Reconstruction Algorithm: Validation Using Ocean Model and In Situ Soil Moisture Data". IEEE Transactions on Geoscience and Remote Sensing, Volume 53, Issue 9, pages: 4961 - 4971.

Lemmetyinen J., J. Uusitalo, J. Kainulainen, K. Rautiainen, N. Fabritius, M. Levander, V. Kangas, H. Greus, J. Pihlflyckt, A. Kontu, S. Kemppainen, A. Colliander, M. Hallikainen, J. Lahtinen, (2007), "SMOS Calibration Subsystem", IEEE Transactions On Geoscience and Remote Sensing, Vol. 45, No. 11, pp. 3691-3700.

Lin W., I. Corbella, F. Torres, N. Duffo, M. Martín-Neira, (2011), "Correction of Spatial Errors in SMOS Brightness Temperature Images”, Proceedings of IGARSS-2011, Vancouver (Canada).

Lin Wu, Torres, F., Corbella, I., Duffo, N., Martín-Neira, M., (2012), "Performance of a spatial error correction technique in SMOS brightness temperature images", MicroRad-2012, Villa Mondragone, Tor Vergata University of Rome (Italy).

Lin W., F. Torres, I. Corbella, N. Duffo, I. Durán, M. Vall-llossera, A. Camps, S. Delwart, M. Martín-Neira, (2013), "Radiometric Performance of SMOS Full Polarimetric Imaging", IEEE Geoscience and Remote Sensing Letters, vol 10, Issue 6, pp 1454-1458. 


\section{REPLACE THIS LINE WITH YOUR PAPER IDENTIFICATION NUMBER (DOUBLE-CLICK HERE TO EDIT) <}

Macelloni, G, Brogioni M., Pettinato, S., Zasso, R. Crepaz, A., Zaccaria, J., Drinkwater, M., (2013), "Ground based L-Band Emission Measurements at DOME-C Antarctica: the DOMEX-2 experiment. IEEE Trans. Geosci. and Remote Sensing, vol 51, No 9, 4718-4730.

Martín-Neira M., S. Ribó, A. J. Martín-Polegre, (2002), “Polarimetric mode of MIRAS,” IEEE Transactions on Geoscience and Remote Sensing, vol. 40, no. 8, pp. 1755-1768.

Martín-Neira M., M. Suess, J. Kainulainen, F. Martín-Porqueras, (2008), "The Flat Target Transformation", IEEE Transactions On Geoscience And Remote Sensing, Vol. 46, No. 3, 613 - 620.

Martin-Porqueras F., J. Kainulainen, M. Martín-Neira, I. Corbella, R. Oliva, R. Castro, J. Barbosa, A. Gutierrez, (2010), "Experimental Validation of the Corbella's Visibility Function using HUT-2D and MIRAS", Proceedings of IGARSS-2010, Honolulu, Hawaii (US), pp.4286-4289.

McMullan K., M. Brown, M. Martín-Neira, W. Rits, S. Ekholm, J. Marti and J. Lemanczyk, (2008), "SMOS: The payload”, IEEE Transactions On Geoscience and Remote Sensing, Vol. 46, No. 3, 594 - 605.

Mecklenburg Susanne, Matthias Drusch, Yann H. Kerr, Senior Member, IEEE, Jordi Font, Manuel Martín-Neira, Steven Delwart, Guillermo Buenadicha, Nicolas Reul, Elena Daganzo-Eusebio, Roger Oliva, and Raffaele Crapolicchio, (2012), "ESA's Soil Moisture and Ocean Salinity Mission: Mission Performance and Operations", IEEE TGARS, Vol 50, No 5, pages 1354-1366.

Mecklenburg S., M. Drusch, Y. Kerr, J. Font, N. Reul, M. Martín-Neira, R. Oliva, E. Daganzo-Eusebio, J. Fauste, L. Kaleschke, N. Rodriguez-Fernandez, P. de Rosnay, G. Macelloni, J. Muñoz-Sabater, K. Rautiainen, J.P. Grant, N. Verhoest, H. Lievens, P. Richaume, R. Sabia, S. Delwart, R. Crapolicchio, A. de la Fuente, M. Kornberg, (in press), "ESA's Soil Moisture and Ocean Salinity Mission: from Science to Operational Applications". This RSE special issue, submitted.

Oliva R., E. Daganzo, Y. Kerr, S. Mecklenburg, S. Nieto, P. Richaume, C. Gruhier, (2012), "SMOS Radio Frequency Interference Scenario: Status and Actions Taken to Improve the RFI Environment in the 1400-1427 MHz Passive Band". IEEE Trans Geosci. Remote Sens., Special issue on Soil Moisture and Ocean Salinity Mission. Vol. 50, No 5, pp. 1427-1439.

Oliva R., M. Martín-Neira, I. Corbella, F. Torres, J. Kainulainen, J.E. Tenerelli, F. Cabot, F. Martin-Porqueras, (2013), "SMOS Calibration and Instrument Performance after One Year in Orbit", IEEE Transactions on Geoscience and Remote Sensing, Vol. 51, No 1, pp. 654-670.

Oliva R., E. Daganzo, Y. Soldo, Y. Kerr, F. Cabot, P. Richaume, E. Anterrieu, A. Gutierrez, J. Barbosa, G. Lopes, (in press), "Status of RFI in the 1400-1427 MHz passive band: The SMOS perspective", This RSE special issue, submitted.

Rubiales P., J. Closa, E. Checa, S. Dolce, M. Kornberg, M. Martín-Neira, (2015), "SMOS Payload Thermal Control: Review of performances after 5 years in orbit operation", $45^{\text {th }}$ International Conference on Environmental Systems, Bellevue, Washington (US).

SMOS Level 2 Ocean Salinity Team, "SMOS L2 Ocean Salinity Algorithm Theoretical Baseline Document", ARGANS, SO-TN-ARG-GS-0007_L2OS-ATBD_v3.12_150731, July 2015.

Torres F., I. Corbella, A. Camps, N. Duffo, M. Vall-llossera, S. Beraza, C. Gutierrez, M. Martín-Neira, (2006), "Denormalization of Visibilities for In-Orbit Calibration of Interferometric Radiometers", IEEE Transactions on Geoscience and Remote Sensing. Vol. 44, No. 10, pp.2679-2686.

Torres F., I. Corbella, L. Wu, N. Duffo, J. Gourrion, J. Font, M. Martín-Neira, (2012), "Minimization of Image Distortion in SMOS Brightness Temperature Maps Over the Ocean", GeoScience and Remote Sensing Letters, Vol. 9, No. 1, pp.18-22. 
REPLACE THIS LINE WITH YOUR PAPER IDENTIFICATION NUMBER (DOUBLE-CLICK

HERE TO EDIT) <

Torres F., I. Corbella, I. Duran, W. Lin, N. Duffo, M. Martín-Neira, (2014), "Residual Calibration Error Impact On SMOS SLL Performance”, IGARSS-2014 Proceedings, pp.2542-2545, Quebec (Canada).

Torres F., I. Corbella, L. Wu, N. Duffo, S. Delwart, M. Martín-Neira, (2015), "In-Orbit Validation of SMOS Full 